\title{
Zeitschriftenverzeichnis nach Abkürzungen
}

\begin{tabular}{|c|c|c|c|}
\hline AA & $=$ Archäologischer Anzeiger & ActaACarp & $=$ Acta archaeologica carpathica \\
\hline AAA & 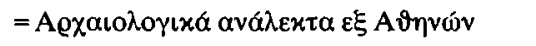 & ActaALov & $=$ Acta archaeologica Lovaniensia \\
\hline AAdv & $=$ The Archaeological Advertiser & ActaAntHung & $=$ Acta antiqua Academiae scientiarum \\
\hline \multirow[t]{2}{*}{$\mathrm{AAJ}$} & \multirow{2}{*}{$\begin{array}{l}=\text { Annual of the Department of Antiquities } \\
\text { of Jordan }\end{array}$} & & hungaricae \\
\hline & & ActaArch & $=$ Acta archaeologica. København \\
\hline AarbKøb & $\begin{array}{l}=\text { Aarboger for nordisk oldkyndighed og } \\
\text { historie }\end{array}$ & ActaArchHung & $\begin{aligned} &= \text { Acta archaeologica Academiae } \\
& \text { scientiarum hungaricae }\end{aligned}$ \\
\hline \multirow{3}{*}{$\begin{array}{l}\text { AAS } \\
\text { AASOR }\end{array}$} & $=$ Annales archéologiques arabes syriennes & \multirow[t]{2}{*}{ ActaCl } & \multirow{2}{*}{$\begin{aligned}= & \text { Acta classica. Proceedings of the Classical } \\
& \text { Association of South Africa }\end{aligned}$} \\
\hline & $=$ The Annual of the American Schools of & & \\
\hline & $\begin{aligned} & \text { Oriental Research } \\
= & \text { Archäologische Ausgrabungen in Baden- }\end{aligned}$ & ActaCIDebrec & $\begin{aligned}= & \text { Acta classica Universitatis scientiarum } \\
& \text { Debreceniensis }\end{aligned}$ \\
\hline AAusgrBadWürt & $\begin{aligned} & \text { Württemberg } \\
= & \text { Archaeologia austriaca }\end{aligned}$ & ActaHistDac & $\begin{aligned}= & \text { Acta historica. Societas academica } \\
& \text { dacoromana }\end{aligned}$ \\
\hline AbhBerlin & $\begin{array}{l}=\text { Abhandlungen der Deutschen Akademie } \\
\text { der Wissenschaften zu Berlin }\end{array}$ & ActaHyp & $\begin{array}{l}=\text { Acta hyperborea. Danish studies in } \\
\text { classical archaeology }\end{array}$ \\
\hline AbhLeipzig & $\begin{array}{l}=\text { Abhandlungen der Sächsischen Akademie } \\
\text { der Wissenschaften zu Leipzig. } \\
\text { Philologisch-historische Klasse }\end{array}$ & $\begin{array}{l}\text { ActalnstRomFin } \\
\text { ActaMusNapoca }\end{array}$ & $\begin{array}{l}=\text { Acta Instituti romani Finlandiae } \\
=\text { Acta Musei Napocensis }\end{array}$ \\
\hline AbhMainz & $\begin{array}{l}=\text { Abhandlungen der Geistes- und } \\
\text { Sozialwissenschaftlichen Klasse. } \\
\text { Akademie der Wissenschaften und der } \\
\text { Literatur in Mainz }\end{array}$ & $\begin{array}{l}\text { ActaMusPorol } \\
\text { ActaNum } \\
\text { ActaOrHung }\end{array}$ & $\begin{aligned}= & \text { Acta Musei Porolissensis } \\
= & \text { Acta numismàtica (Barcelona) } \\
= & \text { Acta orientalia Academiae scientiarum } \\
& \text { hungaricae }\end{aligned}$ \\
\hline \multirow[t]{2}{*}{ AbhMünchen } & \multirow[t]{2}{*}{$\begin{aligned}= & \text { Bayerische Akademie der Wissenschaften. } \\
& \text { Philosophische-historische Klasse. } \\
& \text { Abhandlungen }\end{aligned}$} & ActaPhilSocDac & $\begin{array}{l}=\text { Acta philologica. Societas academica } \\
\text { dacoromana }\end{array}$ \\
\hline & & ActaPraehistA & $=$ Acta praehistorica et archaeologica \\
\hline Ahr-Nahrain & $\begin{array}{l}=\text { Archeologie en Bretagne. Bulletin } \\
\text { d'information }\end{array}$ & ActaTorunA & $\begin{aligned}= & \text { Acta Universitatis Nicolai Copernici. } \\
& \text { Archaeologia }\end{aligned}$ \\
\hline Abr-Nahrain & $\begin{array}{l}=\text { Abr-Nahrain. An annual published by the } \\
\text { Department of Middle Eastern Studies, } \\
\text { University of Melbourne }\end{array}$ & ActaTorunHist & $\begin{array}{l}=\text { Acta Universitatis Nicolai Copernici. } \\
\text { Historia (Torun) }\end{array}$ \\
\hline ACalc & $=$ Archeologia e calcolatori & ADelt & 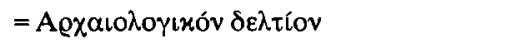 \\
\hline \multirow[t]{3}{*}{ ACamp } & \multirow{3}{*}{$\begin{array}{l}=\text { Archeologia in Campania. Bolletino di } \\
\text { informazioni a cura della Soprintendenza } \\
\text { archeologica delle province di Napoli e } \\
\text { Caserta }\end{array}$} & $\mathrm{AE}$ & = L’année épigraphique \\
\hline & & Aegaeum & $\begin{array}{l}=\text { Aegaeum. Annales d'archéologie égéenne } \\
\text { de l'Université de Liège }\end{array}$ \\
\hline & & AegHelv & $=$ Aegyptiaca helvetica \\
\hline ACant & $=$ Archaeologia Cantiana & Aegyptus & $=$ Aegyptus \\
\hline AcBibl & = Accademie e biblioteche d'Italia & AEphem & 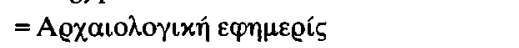 \\
\hline \multirow[t]{2}{*}{ Acme } & \multirow{2}{*}{$\begin{array}{l}=\text { Acme. Annali della Facoltá di lettere e } \\
\text { filosofia dell'Universitá degli studi di } \\
\text { Milano }\end{array}$} & AeR & $=$ Atene $\mathrm{e}$ Roma \\
\hline & & AErgoMak & 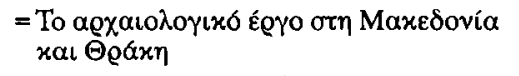 \\
\hline \multirow[t]{2}{*}{ ActaAArtHist } & \multirow{2}{*}{$\begin{array}{l}=\text { Acta ad archaeologiam et artium } \\
\text { historiam pertinentia }\end{array}$} & AErt & = Archaeologiai értesitö \\
\hline & & Aevum & $\begin{array}{l}=\text { Aevum. Rassegna di scienze storiche } \\
\text { linguistiche e filologiche }\end{array}$ \\
\hline ActaAArtHist s. a. & $\begin{aligned}= & \text { Acta ad archaeologiam et artium } \\
& \text { historiam pertinentia. Series altera in } 8^{\circ}\end{aligned}$ & AevumAnt & $=$ Aevum antiquum \\
\hline ActaAcAbo & $=$ Acta Academiae Aboensis & AfO & $=$ Archiv für Orientforschung \\
\hline
\end{tabular}


Africa

AGeo

ÄgLev

AHistStAlex

AInf

AIPhOr

AJA

AJahrBay

$\mathrm{AJPh}$

AJug

AKorrBl

Alba Regia

AlmaMaterSt

AlmanachWien

AlonJisrael

Altamura

Altertum

AltoMed

AltorF

AltThür

AM

AMediev

AMethTh

AMI

AmJAncHist

AmJNum

AMold

AMosel

Ampurias

AmStP

ANachrBad

Anadolu

AnadoluAraş

AnadoluY1l

Anagennesis

AnalBolland

AnalRom

Anas

Anatolica

AnatSt

ANaturwiss

AncHistB

Anclnd

AncSoc

AncWorld
= Africa. Institut national d'archéologie et d'art, Tunis

= Archaeologia geographica

= Ägypten und Levante. Zeitschrift für ägyptische Archäologie und deren Nachbargebiete

$=$ Archaeological and Historical Studies. The Archaeological Society of Alexandria.

$=$ Archäologische Informationen. Mitteilungen zur Ur- und Frühgeschichte

= Annuaire de l'Institut de philologie et d'histoire orientales et slaves

$=$ American Journal of Archaeology

= Das archäologische Jahr in Bayern

= American Journal of Philology

$=$ Archaeologia jugoslavica

= Archäologisches Korrespondenzblatt

= Alba Regia. Annales Musei Stephani Regis

= Alma mater studiorum

= Almanach der Wiener Akademie der Wissenschaften

$=$ 'Alon mahleqat ha- ${ }^{-}$atiqot šel medinat Jisra'el

= Altamura. Bollettino dell'ArchivioBiblioteca-Museo civico

$=$ Das Altertum

$=$ Alto medioevo

$=$ Altorientalische Forschungen

$=$ Alt - Thüringen

$=$ Mitteilungen des Deutschen Archäologischen Instituts, Athenische Abteilung

$=$ Archeologia medievale. Cultura materiale, insediamenti, territorio

$=$ Advances in Archaeological Method and Theory

= Archäologische Mitteilungen aus Iran

$=$ American Journal of Ancient History

= American Journal of Numismatics

$=$ Archeologia moldovei

= Archaeologia Mosellana

$=$ Ampurias

= American Studies in Papyrology

= Archäologische Nachrichten aus Baden

$=$ Anadolu (Anatolia). Ankara

= Anadolu araştırmaları. Jahrbuch für kleinasiatische Forschung

= Anadolu medeniyetleri müzesi yıllığ 1

= Anagennesis. A papyrological journal

= Analecta Bollandiana

= Analecta romana Instituti danici

= Anas. Museo nacional de arte romano de Mérida

$=$ Anatolica

$=$ Anatolian Studies

$=$ Archäologie und Naturwissenschaften

$=$ The Ancient History Bulletin

$=$ Ancient India

$=$ Ancient Society

$=$ The Ancient World
AnDubr

AnEcSoc

ANews

AnFilCl

AnGerona

AnHistDer

ANilMoy

ANL

AnMunFaro

AnMurcia

AnnAcEtr

AnnAcTorino

AnnAStorAnt

AnnBari

AnnBenac

AnnBiblaModena

AnnByzConf

AnnCagl

AnnCaglMag

AnnEconSocCiv

AnnEgBibl

AnnEth

AnnFaina

AnnIstGiapp

AnnIstItNum

AnnLecce

AnnLeedsUnOrSoc

AnnMacerata

AnnMessMag

AnnNap

AnnNivern

AnnOrNap

AnnPerugia

AnnPisa

AnnPontAcRom

AnnRepBrSAth

AnnRepCypr
= Anali Zavoda za povijesne znanosti Istraživačkog centra Jugoslavenske akademije znanosti i umjetnosti u Dubrovniku

= Anuario de historia económica y social

$=$ Archaeological News

$=$ Anales de filología clásica. Universidad de Buenos Aires

$=$ Anales del Instituto de estudios Gerundenses

= Anuario de historia del derecho español

$=$ Archéologie du Nil Moyen

$=$ The Archaeological News Letter

$=$ Anais do município de Faro

$=$ Anales de prehistoria y arqueología. Universidad de Murcia

= Annuario. Accademia etrusca di Cortona

= Annuario della Accademia delle scienze di Torino

$=$ Annali del Seminario di studi del mondo classico, Sezione di archeologia e storia antica. Napoli

$=$ Annali della Facoltà di lettere $\mathrm{e}$ filosofia. Università Bari

$=$ Annali Benacensi

= Annuario bibliografico di archeologia

= Annual Byzantine Studies Conference. Abstracts of Papers

= Annali della Facoltà di lettere e filosofia della Università di Cagliari

$=$ Annali della Facoltà di magistero dell'Università di Cagliari

= Annales. Economies, sociétés, civilisations

= Annual Egyptological Bibliography

$=$ Annales d'Ethiopie

= Annali della Fondazione per il Museo "Claudio Faina"

$=$ Annuario. Istituto giapponese di cultura in Roma

$=$ Annali. Istituto italiano di numismatica

= Annali dell'Università di Lecce. Facoltà di lettere e filosofia e di magistero

$=$ The Annual of Leeds University Oriental Society

= Annali della Facoltà di lettere e filosofia. Università di Macerata

= Nuovi annali della Facoltà di magistero dell'Università di Messina

= Annali della Facoltà di lettere e filosofia, Università di Napoli

$=$ Les annales des Pays Nivernais

$=$ Annali. Istituto orientale di Napoli

= Annali della Facoltà di lettere e filosofia, Università degli studi di Perugia

$=$ Annali della Scuola normale superiore di Pisa

$=$ Annuario della Pontificia accademia romana di archeologia

$=$ The British School of Archaeology at Athens. Annual report of the managing comittee

$=$ Annual Report of the Director of the Department of Antiquities, Republic of Cyprus 


\begin{tabular}{|c|c|c|c|}
\hline \multirow[t]{2}{*}{ AnnRepFoggArtMus } & \multirow{2}{*}{$\begin{aligned}= & \text { The Annual Report of the Fogg Art } \\
& \text { Museum }\end{aligned}$} & Archaeonautica & $=$ Archaeonautica \\
\hline & & Archaiognosia & $=A \varrho \chi \alpha \iota \gamma v \omega \sigma i \alpha$ \\
\hline AnnuarioAcLinc & $\begin{aligned}= & \text { Annuario della Accademia nazionale dei } \\
& \text { Lincei }\end{aligned}$ & Archäographie & $\begin{aligned}= & \text { Archäographie. Archäologie und } \\
& \text { elektronische Datenverarbeitung }\end{aligned}$ \\
\hline AnnuarioLecce & $\begin{aligned}= & \text { Annuario. Liceo-ginnasio statale } \mathrm{G} . \\
& \text { Palmieri, Lecce }\end{aligned}$ & ArchBegriffsGesch & $=$ Archiv für Begriffsgeschichte \\
\hline \multirow[t]{2}{*}{ AnnUnBud } & \multirow{2}{*}{$\begin{aligned}= & \text { Annales Universitatis scientiarum } \\
& \text { Budapestinensis de Rolando Eötvös } \\
& \text { nominatae. Sectio classica }\end{aligned}$} & & 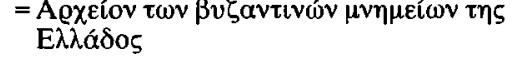 \\
\hline & & ArchCl & $=$ Archeologia classica \\
\hline AnnWorcArtMus & $=$ Annual (Report). Worcester Art Museum & Archeo & $=$ Archeo. Attualità del passato \\
\hline \multirow[t]{3}{*}{ Antaeus } & \multirow{3}{*}{$\begin{array}{l}=\text { Antaeus. Mitteilungen des } \\
\text { Archäologischen Instituts der } \\
\text { Ungarischen Akademie der } \\
\text { Wissenschaften }\end{array}$} & Archeogr'Triest & $=$ Archeografo triestino \\
\hline & & ArcheologiaParis & $=$ Archeologia. Trésor des âges \\
\hline & & ArcheologiaRoma & $=$ Archeologia. Rivista bimestrale. Roma \\
\hline AntAfr & $=$ Antiquités africaines & ArcheologiaWarsz & $\begin{aligned}= & \text { Archeologia } . \text { Rocznik Instytutu historii } \\
& \text { kultury materialnej Polskiej akademii }\end{aligned}$ \\
\hline AntCl & = L'antiquité classique & & nauk \\
\hline AnthrAChron & 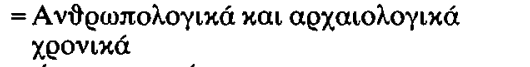 & ArcheologijaKiiv & $\begin{array}{l}=\text { Archeologija. Akademija nauk } \\
\text { Ukrains'koi RSR, Institut archeologii }\end{array}$ \\
\hline Anthropos & 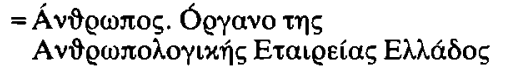 & ArcheologijaSof & $\begin{aligned} &= \text { Archeologija. Organ na Archeologičeskij } \\
& \text { institut i muzej pri Bălgarskata akademija }\end{aligned}$ \\
\hline \multirow[t]{2}{*}{ Antichthon } & \multirow{2}{*}{$\begin{array}{l}=\text { Antichthon. Journal of the Australian } \\
\text { Society for Classical Studies }\end{array}$} & & na naukite \\
\hline & & Arc & $=$ Archivo español de arqueología \\
\hline Antipolis & $\begin{array}{l}=\text { Antipolis. A Journal of Mediterranean } \\
\text { Archaeology }\end{array}$ & ArchEv & 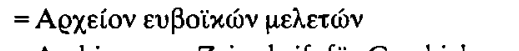 \\
\hline \multirow[t]{2}{*}{ Antiqua } & \multirow{2}{*}{$\begin{array}{l}\text { = Antiqua. Pubblicazione bimestrale } \\
\text { dell'Archeoclub d'Italia per la } \\
\text { conoscenza, la tutela e la valorizzazione } \\
\text { dei beni culturali }\end{array}$} & Arc & $\begin{array}{l}=\text { Architectura. Zeitschrift für Geschichte } \\
\text { der Baukunst }\end{array}$ \\
\hline & & ArchPF & $\begin{aligned}= & \text { Archiv für Papyrusforschung und } \\
& \text { verwandte Gebiete }\end{aligned}$ \\
\hline Antiquity & $=$ Antiquity & ArchPrehistLev & $=$ Archivo de prehistoria levantina \\
\hline AntJ & $\begin{array}{l}=\text { The Antiquaries Journal } \\
=\text { Antike Kunst }\end{array}$ & ArchStorCal & $\begin{array}{l}=\text { Archivio storico per la Calabria e la } \\
\text { Lucania }\end{array}$ \\
\hline \multirow[t]{2}{*}{ AntNat } & \multirow{2}{*}{$\begin{array}{l}=\text { Antiquités nationales. Saint-Germain-en- } \\
\text { Laye }\end{array}$} & ArchStorPugl & $=$ Archivio storico pugliese \\
\hline & & ArchStorRom & $=$ Archivio della Società romana di storia \\
\hline AntPisa & = Antichità Pisane & & \\
\hline AntSurv & $=$ Antiquity and Survival & ArchStorSicOr & $=$ Archivio storico per la Sicilia orientale \\
\hline AnzAW & $=$ Anzeiger für die Altertumswissenschaft & Arctos & $=$ Arctos. Acta philologica fennica \\
\hline AnzWien & $\begin{aligned}= & \text { Anzeiger. Österreichische Akademie der } \\
& \text { Wissenschaften, Wien }\end{aligned}$ & $\begin{array}{l}\text { ARepLond } \\
\text { Arethusa }\end{array}$ & $\begin{array}{l}=\text { Archaeological Reports } \\
=\text { Arethusa }\end{array}$ \\
\hline APamKiiv & $=$ Archeologični pamjatki URSR & Argo & $=$ Argo. Narodni muzej v Ljubljani \\
\hline APol & $=$ Archaeologia polona & Arion & $=$ Arion \\
\hline Apollo & $\begin{aligned}= & \text { Apollo. Bollettino dei musei provinciali } \\
& \text { del Salernitano }\end{aligned}$ & ArOr & = Archív orientální \\
\hline ApolloLond & \multirow{2}{*}{$\begin{array}{l}=\text { Apollo. The international magazine of the } \\
\text { arts }\end{array}$} & ARc & $=$ Archeolog \\
\hline \multirow{2}{*}{$\begin{array}{l}\text { APort } \\
\text { APregl }\end{array}$} & & eja & $\begin{array}{l}\text { = Arquivo de Beja. Boletim, estudos, } \\
\text { arquivo }\end{array}$ \\
\hline & $\begin{aligned}= & \text { O arqueólogo português } \\
= & \text { Arheološki pregled. Arheološko društvo } \\
& \text { Jugoslavije }\end{aligned}$ & Arqueologi & $\begin{aligned}= & \text { Arqueología. Memoria de las actuaciones } \\
& \text { programadas en el año } 19 \mathrm{xx}\end{aligned}$ \\
\hline \multirow[b]{2}{*}{ Aquileia chiama } & $=$ Apulum. Acta Musei Apulensis & Arsberättelse & $=$ Arsberättelse \\
\hline & $\begin{aligned}= & \text { Aquileia chiama. Bollettino trimestrale } \\
& \text { della Associazione nazionale per Aquileia }\end{aligned}$ & Arse & $\begin{array}{l}=\text { Arse. Boletín del Centro arqueológico } \\
\text { Saguntino }\end{array}$ \\
\hline AquilNost & $=$ Aquileia nostra & ArsGeorg & $=$ Ars georgica \\
\hline Aquitania & $\begin{array}{l}=\text { Aquitania. Une revue inter-régionale } \\
\text { d'archéologie }\end{array}$ & $\begin{array}{l}\text { ArtAntMod } \\
\text { ArtARhône }\end{array}$ & $\begin{array}{l}=\text { Arte antica e moderna } \\
=\text { Art et archéologie en Rhône-Alpes }\end{array}$ \\
\hline ArabAEpigr & $=$ Arabian Archaeology and Epigraphy & ArtAs & $=$ Artibus Asiae \\
\hline ARadRaspr & $=$ Arheološki radovi i rasprave & ArtB & $=$ The Art Bulletin \\
\hline ArbFBerSächs & $\begin{array}{l}=\text { Arbeits- und Forschungsberichte zur } \\
\text { sächsischen Bodendenkmalpflege }\end{array}$ & Art] & $=$ Art Journal \\
\hline \multirow[t]{2}{*}{ Archaeologia } & \multirow{2}{*}{$\begin{aligned}= & \text { Archaeologia or Miscellaneous Tracts } \\
& \text { Relating to Antiquity Published by the } \\
& \text { Society of Antiquaries of London }\end{aligned}$} & $\begin{array}{l}\text { ArtLom } \\
\text { ArtVirg }\end{array}$ & $\begin{array}{l}=\text { Arte lombarda } \\
=\text { Arts in Virginia }\end{array}$ \\
\hline & & ASAE & $=$ Annales du Service des antiquités de \\
\hline \multirow{3}{*}{$\begin{array}{l}\text { Archaeology } \\
\text { Archaeometry }\end{array}$} & $=$ Archaeology. A magazine dealing with & & \\
\hline & & & $\begin{aligned}= & \text { Annuario della Scuola archeologica } \\
& \text { Atene e delle Missioni italiane in Or }\end{aligned}$ \\
\hline & $\begin{array}{l}\text { Laboratory for Archaeology and History } \\
\text { of Art. Oxford University }\end{array}$ & ASbor & $\begin{aligned}= & \text { Archeologičeskij sbornik. } \\
& \text { Gosudarstvennyj ordena Lenina Ermitaž }\end{aligned}$ \\
\hline
\end{tabular}


ASchw

ASoc

ASoloth

Assaph

AssyrMisc

Athenaeum

'Atiqot

Atlal

AttiAcPontan

AtriAcRov

AttiAcTorino

AttiCAntCl

AttiMemBologna

AttiMemDal

AttiMemFirenze

AttiMemIstria

AttiMemMagnaGr

AtriMemModena

AttiMemTivoli

AttiMusTrieste

AttiPalermo

AttiRovigno

AttiSocFriuli

AttiVenezia

AuA

AulaOr

Ausa

AusgrFu

AusgrFuWestf

AustrRom

AVen

AVes

AViva

AW

AyasofyaMüzYll
= Archäologie der Schweiz.

Mitteilungsblatt der Schweizerischen

Gesellschaft für Ur- und Frühgeschichte

$=$ Archeologia e società

$=$ Archäologie des Kantons Solothurn

= Assaph. Studies in Art History

= Assyriological Miscellanies

$=$ Athenaeum. Studi periodici di letteratura e storia dell'antichità. Università di Pavia

$=$ 'Atiqot. English series

= Atlal. The journal of Saudi Arabian archaeology

= Atti della Accademia Pontaniana

= Atti della Accademia Roveretana degli Agiati. Contributi della classe di scienze umane, di lettere ed arti.

= Atti della Accademia delle scienze di Torino

$=$ Atti. Centro ricerche e documentazione sull'antichità classica

$=$ Atti e memorie. Deputazione di storia patria per le province di Romagna

= Atti e memorie della Società dalmata di storia patria

= Atti e memorie dell'Academia toscana di scienze e lettere "La Colombaria“"

= Atti e memorie della Società istriana di archeologia e storia patria

= Atti e memorie della Società Magna Grecia

$=$ Atti e memorie. Deputazione di storia patria per le antiche provincie modenesi

$=$ Atti e memorie della Società tiburtina di storia e d'arte

$=$ Atti dei Civici musei di storia ed arte di Trieste. Atti del Museo civico di antichità

$=$ Atti della Accademia di scienze, lettere e arti di Palermo

$=$ Atti. Centro di ricerche storiche, Rovigno

$=$ Atti della Società per la preistoria $\mathrm{e}$ protostoria della regione Friuli - Venezia Giulia

$=$ Atti. Istituto veneto di scienze, lettere ed arti

$=$ Antike und Abendland

$=$ Aula orientalis. Revista de estudios del Próximo Oriente antiguo

$=$ Ausa (Vic)

$=$ Ausgrabungen und Funde. Nachrichtenblatt für Vor- und Frühgeschichte

= Ausgrabungen und Funde in WestfalenLippe

= Pro Austria romana

$=$ Archeologia veneta

$=$ Arheološki vestnik (Ljubljana)

$=$ Archeologia viva

=Antike Welt. Zeitschrift für Archäologie und Kulturgeschichte

= Ayasofia müzesi yıllığı. Annual of Ayasofya Museum
BA

BAAlger

BAAntNat

BABarcel

BABesch

BAcHist

BACopt

BadFuBer

Baetica

BajoAr

Balcanoslavica

BalkSt

BALond

$\mathrm{BaM}$

BAMaroc

BAmSocP

BAncOrMus

BAngers

BAngloIsrASoc

BAnnMusFerr

BAntFr

BAntLux

BAParis

BAProv

BArchAlex

BArchit

BASard

BAsInst

BASOR

BAssBudé

BAssMosAnt

BASudEstEur

BATarr

BAur

Bay VgBl

BBaltimore

BBasil

BBelArt
$=$ Bollettino di archeologia

= Bulletin d'archéologie algérienne

$=$ Bulletin archéologique du Comité des travaux historiques et scientifiques. Antiquités nationales

$=$ Butlletí informatiu de l'Institut de prehistòria i arqueologia de la Diputació provincial de Barcelona

$=$ Bulletin van de Vereeniging tot bevordering der kennis van de antieke beschaving te 's-Gravenhage

= Boletín de la Real academia de la historia

= Bulletin de la Société d'archéologie copte

$=$ Badische Fundberichte

= Baetica. Estudios de arte, geografía e historia

= Bajo Aragón. Prehistoria

= Balcanoslavica

= Balkan Studies

= Bulletin of the Institute of Archaeology, University of London

$=$ Baghdader Mitteilungen

$=$ Bulletin d'archéologie marocaine

$=$ The Bulletin of the American Society of Papyrologists

$=$ Bulletin of the Ancient Orient Museum (Tokyo)

$=$ Bulletin du Centre de recherches et d'enseignement de l'antiquité, Angers

$=$ Bulletin of the Anglo-Israel Archaeological Society

$=$ Bollettino annuale. Musei ferraresi

$=$ Bulletin de la Société nationale des antiquaires de France

$=$ Bulletin des antiquités luxembourgeoises

= Bulletin archéologique du Comité des travaux historiques et scientifiques

$=$ Bulletin archéologique de Provence

=Bulletin. Société archéologique d'Alexandrie

$=$ Bollettino del Centro di studi per la storia dell'architettura

$=$ Nuovo Bullettino archeologico sardo

$=$ Bulletin of the Asia Institute

$=$ Bulletin of the American Schools of Oriental Research

$=$ Bulletin de l'Association Guillaume Budé

$=$ Bulletin d'information de l'Association internationale pour l'étude de la mosaïque antique

= Bulletin d'archéologie sud-est européenne

= Boletín arqueológico. Real sociedad arqueológica tarragonese

$=$ Boletín Auriense

$=$ Bayerische Vorgeschichtsblätter

= The Walters Art Gallery Bulletin

$=$ Bollettino storico della Basilicata

$=$ Boletín de bellas artes. Sevilla 


\begin{tabular}{|c|c|c|c|}
\hline BBelgRom & $\begin{array}{l}=\text { Bulletin de l'Institut historique belge de } \\
\text { Rome }\end{array}$ & BFilLingSic & $\begin{aligned} &= \text { Bollettino. Centro di studi filologici e } \\
& \text { linguistici siciliani }\end{aligned}$ \\
\hline BBurgos & $\begin{aligned}= & \text { Boletín de la Institución Fernán } \\
& \text { Gonzáles, Burgos }\end{aligned}$ & $\begin{array}{l}\text { BFoligno } \\
\text { BiblA }\end{array}$ & $\begin{array}{l}=\text { Bollettino storico della città di Foligno } \\
=\text { The Biblical Archaeologist }\end{array}$ \\
\hline BCamuno & $\begin{aligned} &= \text { Bullettino del Centro Camuno di studi } \\
& \text { preistorici }\end{aligned}$ & $\begin{array}{l}\text { BiblClOr } \\
\text { Biblos-Press }\end{array}$ & $\begin{array}{l}=\text { Bibliotheca classica orientalis } \\
=\text { Biblos-Press }\end{array}$ \\
\hline BCASic & $=$ Beni culturali $e$ ambientali. Sicilia & BIBulg & = Izvestija na Arheologičeskija institut. \\
\hline BCercleNum & $=$ Bulletin. Cercle d'études numismatiques & & $\begin{array}{l}\text { Bulletin de l'Institut archéologique } \\
\text { bulgare }\end{array}$ \\
\hline $\mathrm{BCH}$ & = Bulletin de correspondance hellénique & RICS & $\begin{aligned} & \text { bulgare } \\
= & \text { Bulletin. Institute of Classical Studies, }\end{aligned}$ \\
\hline BCircNumNap & $\begin{aligned} &= \text { Bollettino del Circolo numismatico } \\
& \text { napoletano }\end{aligned}$ & BICS & University of London \\
\hline $\mathrm{BCl}$ & $=$ Bollettino dei classici & BIFAO & $\begin{array}{l}=\text { Bulletin de l'Institut français } \\
\text { d'archéologie orientale }\end{array}$ \\
\hline BClevMus & $\begin{aligned} &= \text { The Bulletin of the Cleveland Museum of } \\
& \text { Art }\end{aligned}$ & BInfCentumcellae & $\begin{array}{l}=\text { Bollettino di informazioni. Associazione } \\
\text { archeologica "Centumcellae“ }\end{array}$ \\
\hline BCom & $\begin{aligned} &= \text { Bullettino della Commissione } \\
& \text { archeologica comunale di Roma }\end{aligned}$ & BInstEstHel & $\begin{array}{l}\text { = Boletín del Istituto de estudios helénicos. } \\
\text { Universidad de Barcelona, Facoltad de }\end{array}$ \\
\hline BCord & $\begin{aligned}= & \text { Boletín de la Real academia de Córdoba } \\
& \text { de ciencias, bellas letras y nobles artes }\end{aligned}$ & BiogrZbor & $\begin{aligned} & \text { filosofía y letras } \\
= & \text { Biogradski zbornik }\end{aligned}$ \\
\hline $\mathrm{BdA}$ & $=$ Bollettino d'arte & $\mathrm{BiOr}$ & $=$ Bibliotheca orientalis \\
\hline BDirRom & $\begin{aligned}= & \text { Bullettino dell'Istituto di diritto romano } \\
& \text { "Vittorio Scialoja“" }\end{aligned}$ & BJaén & $\begin{array}{l}=\text { Boletín del Instituto de estudios } \\
\text { giennenses }\end{array}$ \\
\hline BEcAntNimes & $\begin{array}{l}=\text { Bulletin (annuel) de l'Ecole antique de } \\
\text { Nimes }\end{array}$ & BJb & $\begin{array}{l}=\text { Bonner Jahrbücher des Rheinischen } \\
\text { Landesmuseums in Bonn und des Vereins } \\
\text { von Altertumsfreunden im Rheinlande }\end{array}$ \\
\hline BediKart & = Bedi Kartlisa. Revue de kartvélologie & BJerus & $=$ Bulletin. The Hebrew University, \\
\hline BEgNewYork & $\begin{aligned}= & \text { Bulletin of the Egyptological Seminar } \\
& \text { (New York) }\end{aligned}$ & BLaborMusLouvre & $\begin{aligned} & \text { Jerusalem, Rabinowitz Fund } \\
= & \text { Bulletin du laboratoire du Musée du }\end{aligned}$ \\
\hline BeitrAllgA & $\begin{array}{l}=\text { Beiträge zur allgemeinen und } \\
\text { vergleichenden Archäologie }\end{array}$ & BLazioMerid & $\begin{array}{l}\text { Louvre } \\
=\text { Bollettino dell'Istituto di storia e di arte }\end{array}$ \\
\hline BeitrNamF & $=$ Beiträge zur Namenforschung & & del Lazio meridionale \\
\hline BeitrSudanF & $=$ Beiträge zur Sudanforschung & BLikUm & = Bulletin Zavoda za likovne umjetnosti \\
\hline BelArt & $=$ Bellas artes (Madrid) & & umjetnosti \\
\hline Belleten & $=$ Belleten. Türk tarih kurumu & BlMüFreundeF & = Blätter für Münzfreunde und \\
\hline Benàcus & $\begin{array}{l}=\text { Benàcus. Museo archeologico della Val } \\
\text { Tenesi }\end{array}$ & BLugo & $\begin{aligned} & \text { Münzforschung } \\
= & \text { Boletín de la Comisíon provincial de }\end{aligned}$ \\
\hline BerBayDenkmPfl & $\begin{aligned}= & \text { Bericht der Bayerischen } \\
& \text { Bodendenkmalpflege }\end{aligned}$ & & $\begin{array}{l}\text { monumentos históricos y artísticos de } \\
\text { Lugo }\end{array}$ \\
\hline Berceo & $=$ Berceo (Logroño) & BMetrMus & $\begin{array}{l}=\text { The Metropolitan Museum of Art } \\
\text { Bulletin }\end{array}$ \\
\hline BerDF & $\begin{aligned}= & \text { Bericht der Deutschen } \\
& \text { Forschungsgemeinschaft }\end{aligned}$ & BMon & $=$ Bulletin monumental \\
\hline BerlBeitrArchäom & = Berliner Beiträge zur Archäometrie & BMonMusPont & $\begin{array}{l}=\text { Bollettino. Monumenti, musei e gallerie } \\
\text { pontificie }\end{array}$ \\
\hline BerlBlVFrühGesch & $\begin{array}{l}=\text { Berliner Blätter für Vor- und } \\
\text { Frühgeschichte }\end{array}$ & $\begin{array}{l}\text { BMQ } \\
\text { BMusBeyrouth }\end{array}$ & $\begin{array}{l}=\text { The British Museum Quarterly } \\
=\text { Bulletin du Musée de Beyrouth }\end{array}$ \\
\hline BerlJbVFrühGesch & $\begin{array}{l}=\text { Berliner Jahrbuch für Vor- und } \\
\text { Frühgeschichte }\end{array}$ & BMusBrux & $\begin{array}{l}=\text { Bulletin des Musées royaux d'art et } \\
\text { d'histoire, Bruxelles }\end{array}$ \\
\hline BerlMus & $=$ Berliner Museen & BMusCadiz & = Boletín del Museo de Cádiz \\
\hline BerlNumZ & $=$ Berliner numismatische Zeitschrift & BMusCivRom & = Bullettino del Museo della civiltà romana \\
\hline BerOudhBod & $\begin{array}{l}=\text { Berichten van de Rijksdienst voor het } \\
\text { oudheidkundig bodemonderzoek }\end{array}$ & $\begin{array}{l}\text { BMusFA } \\
\text { BMusHongr }\end{array}$ & $\begin{array}{l}=\text { Bulletin. Museum of Fine Arts, Boston } \\
=\text { Bulletin du Musée hongrois des beaux- }\end{array}$ \\
\hline BerRGK & $\begin{aligned}= & \text { Bericht der Römisch-germanischen } \\
& \text { Kommission }\end{aligned}$ & BMusMadr & $\begin{aligned} & \text { arts } \\
= & \text { Boletín del Museo arqueológico nacional }\end{aligned}$ \\
\hline BerVerhLeipz & $\begin{aligned} \text { = Berichte über die Verhandlungen der } \\
\text { Sächsischen Akademie der } \\
\text { Wissenschaften zu Leipzig }\end{aligned}$ & BMusMonaco & $\begin{array}{l}=\text { Bulletin du Musée d'anthropologie } \\
\text { préhistorique de Monaco } \\
=\text { Bollettino del Museo civico di Padova }\end{array}$ \\
\hline Berytus & = Berytus. Archaeological Studies & BMusRom & = Bollettino dei Musei comunali di Roma \\
\hline BEspA & $\begin{aligned}= & \text { Boletín de la Asociación española de } \\
& \text { amigos de la arqueología }\end{aligned}$ & $\begin{array}{l}\text { BMusVars } \\
\text { BMusZaragoza }\end{array}$ & $\begin{array}{l}=\text { Bulletin du Musée national de Varsovie } \\
=\text { Museo de Zaragoza. Boletín }\end{array}$ \\
\hline BEspOr & $\begin{aligned}= & \text { Boletín de la Asociación española de } \\
& \text { orientalistas }\end{aligned}$ & BNumParis & $\begin{array}{l}=\text { Bulletin de la Société française de } \\
\text { numismatique }\end{array}$ \\
\hline BEtOr & $=$ Bulletin d'études orientales & BNumRoma & $=$ Bollettino di numismatica \\
\hline BFilGrPadova & $\begin{aligned}= & \text { Bollettino dell'Istituto di filologia greca, } \\
& \text { Università di Padova }\end{aligned}$ & BOntMus & $\begin{aligned}= & \text { Bulletin of the Royal Ontario Museum of } \\
& \text { Archaeology. University of Toronto }\end{aligned}$ \\
\hline
\end{tabular}


Boreas

BPeintRom

BPI

BPréhistAlp

BProAvent

BProvidence

BracAug

BremABl

BRest

Brigantium

Britannia

BrMusYearbook

BSA

BSiena

BSocAChamp

BSocBiblReinach

BSR

BStLat

BStorArt

BTextilAnc

BTorino

BTravTun

BudReg

BulletinMalibu

BulletinNorthampton
BVallad

BVitoria

BWPr

Byzantina

Byzantino-Bulgarica

Byzantinoslavica

Byzantion

ByzF

ByzJb

ByzZ

Caesaraugusta

Caesarodunum

XIV
= Boreas. Münstersche Beiträge zur Archäologie

$=$ Bulletin de liaison. Centre d'études des peintures murales romaines

= Bullettino di paletnologia italiana

= Bulletin d'études préhistoriques alpines

$=$ Bulletin de l'Association Pro Aventico

= Bulletin of the Rhode Island School of

Design. Museum notes

= Bracara Augusta. Revista cultural da

Camara municipal de Braga

= Bremer archäologische Blätter

$=$ Bollettino dell'Istituto centrale del restauro

= Brigantium. Museo arqueolóxico e histórico

= Britannia. A journal of Romano-British and kindred studies

$=$ The British Museum Yearbook

$=$ The Annual of the British School at Athens

$=$ Bullettino senese di storia patria

= Bulletin de la Société archéologique champenoise

$=$ Bulletin de liaison de la Société des amis de la Bibliothèque Salomon Reinach

$=$ Papers of the British School at Rome

$=$ Bollettino di studi latini

$=$ Bollettino della Unione storia ed arte

$=$ Bulletin du Centre international d'étude des textiles anciens

$=$ Bollettino della Società piemontese di archeologia e belle arti

= Bulletin des travaux de l'Institut national d'archéologie et d'art (Tunis). Comptes rendus

$=$ Budapest régiségei

= Bulletin. J. Paul Getty Museum of Art (Malibu)

$=$ Bulletin. Smith College Museum of Art

$=$ Boletín del Seminario de estudios de arte y arqueología, Universidad de Valladolid

= Boletín de la Institución "Sancho el Sabio"

= Winckelmannsprogramm der Archäologischen Gesellschaft zu Berlin

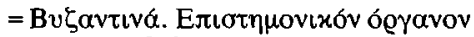

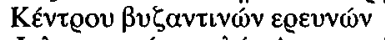

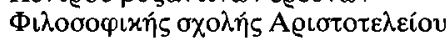

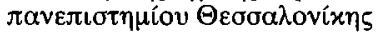

$=$ Byzantino-bulgarica

= Byzantinoslavica

$=$ Byzantion

= Byzantinische Forschungen.

Internationale Zeitschrift für Byzantinistik

= Byzantinisch-neugriechische Jahrbücher

= Byzantinische Zeitschrift

= Caesaraugusta. Publicaciones del Seminario de arqueología y numismática aragonesas

$=$ Caesarodunum

\section{CahArmeeRom \\ CahASubaqu \\ CahByrsa \\ CahCerEg \\ CahDelFrIran \\ CahKarnak \\ CahLig}

CahMariemont

CahMusChampollion

CahPEg

CahRhodBord

CahTun

CalifStClAnt

Capitolium

CArch

Carinthia I

CarnuntumJb

Carpica

Carrobbio

CEDAC

Celticum

CercNum

Chiron

ChronEg

Ciceroniana

CincArtB

CIstAMilano

CivClCr

CivPad

ClAnt

Clio

ClJ

ClMediaev

ClPhil

CIQ

ClR

CMatAOr

Coin Hoards

ColloquiSod
= Cahiers du Groupe de recherches sur l'armée romaine et les provinces

= Cahiers d'archéologie subaquatique

$=$ Cahiers de Byrsa

= Cahiers de la céramique égyptienne

= Cahiers de la Délégation française en Iran

= Cahiers de Karnak

$=$ Cahiers ligures de préhistoire et d'archéologie

$=$ Les cahiers de Mariemont

= Cahiers du Musée Champollion. Histoire et archéologie

= Cahiers de recherches de l'Institut de papyrologie et d'égyptologie de Lille. Sociétés urbaines en Egypte et au Soudan

$=$ Cahiers rhodaniens

$=$ Cahiers de Tunisie

= California Studies in Classical Antiquity

$=$ Capitolium

= Cahiers archéologiques

$=$ Carinthia I. Geschichtliche und volkskundliche Beiträge zur Heimatkunde Kärntens

$=$ Carnuntum-Jahrbuch. Zeitschrift für Archäologie und Kulturgeschichte des Donauraumes

= Carpica. Muzeul judeţean de istorie şi artă, Bacău

= Il Carrobbio. Rivista di studi bolognesi

$=$ CEDAC. Bulletin. Centre d'études et de documentation archéologique de la Conservation de Carthage

= Celticum. Supplément à Ogam

= Cercetări numismatice. Muzeul naţional de istorie

$=$ Chiron. Mitteilungen der Kommision für Alte Geschichte und Epigraphik des Deutschen Archäologischen Instituts

$=$ Chronique d'Egypte

$=$ Ciceronian

$=$ The Cincinnati Art Museum Bulletin

$=$ Contributi dell'Istituto di archeologia. Pubblicazioni dell'Università cattolica del Sacro Cuore, Milano

= Civiltà classica e cristiana

=Civiltà padana. Archeologia e storia del territorio

$=$ Classical Antiquity

$=$ Clio. Revista do Centro de história da Universidade de Lisboa

$=$ The Classical Journal

$=$ Classica et mediaevalia. Revue danoise de philologie et d'histoire

= Classical Philology

$=$ The Classical Quarterly

$=$ The Classical Review

$=$ Contributi e materiali di archeologia orientale

$=$ Coin Hoards. The Royal Numismatic Society, London

$=$ Colloqui del sodalizio 


\begin{tabular}{|c|c|c|c|}
\hline CommunicAHung & $\begin{array}{l}=\text { Communicationes archaeologicae } \\
\text { hungaricae }\end{array}$ & DossAlet & $\begin{aligned}= & \text { Les dossiers du Centre régional } \\
& \text { archéologique d'Alet }\end{aligned}$ \\
\hline CommunicReiCret & $\begin{array}{l}=\text { Communicationes. Rei cretariae romanae } \\
\text { fautores }\end{array}$ & $\begin{array}{l}\text { DossAParis } \\
\text { Durius }\end{array}$ & $\begin{array}{l}=\text { Les dossiers de l'archéologie } \\
=\text { Durius. Boletín castellano de estudios }\end{array}$ \\
\hline Conimbriga & $=$ Conimbriga & & clásicos \\
\hline Conoscenze & $\begin{array}{l}\text { = Conoscenze. Rivista annuale della } \\
\text { Soprintendenza archeologica e per i beni } \\
\text { ambientali, architettonici, artistici e } \\
\text { storici del Molise }\end{array}$ & & \\
\hline Corduba & = Corduba. Museo arqueológico nacional & $\begin{array}{l}\text { EastWest } \\
\text { Eburodunum }\end{array}$ & $\begin{array}{l}=\text { East and West } \\
=\text { Eburodunum }\end{array}$ \\
\hline CRAI & $\begin{array}{l}=\text { Comptes rendus des séances de } \\
\text { l'Académie des inscriptions et belles- } \\
\text { lettres }\end{array}$ & EchosCl & $\begin{array}{l}=\text { Echos du monde classique. Classical } \\
\text { views }\end{array}$ \\
\hline CretSt & $=$ Cretan Studies & Egnatia & 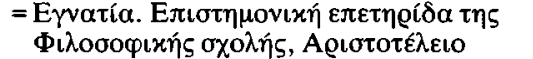 \\
\hline CronAStorArt & $\begin{array}{l}=\text { Cronache di archeologia e di storia } \\
\text { dell'arte }\end{array}$ & & 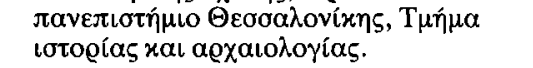 \\
\hline CronErcol & $\begin{aligned}= & \text { Cronache ercolanesi. Bollettino del } \\
& \text { Centro internazionale per lo studio dei } \\
& \text { papiri ercolanesi }\end{aligned}$ & $\begin{array}{l}\text { EgVicOr } \\
\text { Eikasmos }\end{array}$ & $\begin{array}{l}=\text { Egitto e Vicino Oriente } \\
=\text { Eıxaouós. Quaderni bolognesi di } \\
\text { filologia classica }\end{array}$ \\
\hline CronPomp & $=$ Cronache pompeiane & Eirene & $=$ Eirene. Studia graeca et latina \\
\hline CuadCastellon & $\begin{array}{l}=\text { Cuadernos de prehistoria y arqueología } \\
\text { Castellonense }\end{array}$ & Elenchos & $\begin{array}{l}=\text { Elenchos. Rivista di studi sul pensiero } \\
\text { antico }\end{array}$ \\
\hline CuadFilCl & $\begin{array}{l}=\text { Cuadernos de filología clásica. Facultad } \\
\text { de letras y filosofia, Universidad de } \\
\text { Madrid }\end{array}$ & $\begin{array}{l}\text { Ellenika } \\
\text { Emerita }\end{array}$ & 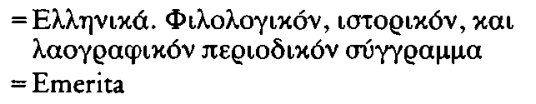 \\
\hline CuadGallegos & $=$ Cuadernos de estudios gallegos & EmPrerom & = Emilia preromana \\
\hline CuadGranada & $\begin{aligned}= & \text { Cuadernos de prehistoria de la } \\
& \text { Universidad de Granada }\end{aligned}$ & $\begin{array}{l}\text { Empúries } \\
\text { Enchoria }\end{array}$ & $\begin{array}{l}=\text { Empúries } \\
=\text { Enchoria. Zeitschrift für Demotistik und }\end{array}$ \\
\hline CuadPrehistA & $=$ Cuadernos de prehistoria y arqueología & & Koptologie \\
\hline CuadRom & $\begin{aligned}= & \text { Cuadernos de trabajos de la Escuela } \\
& \text { española de historia y arqueología en }\end{aligned}$ & Eos & $\begin{array}{l}=\text { Eos. Commentarii Societatis philologae } \\
\text { Polonorum }\end{array}$ \\
\hline & Roma & EpetBoiotMel & 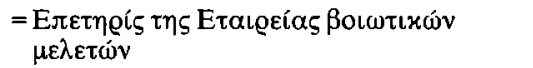 \\
\hline Cypsela & $=$ Cypsela (Girona) & EpetByzSpud & 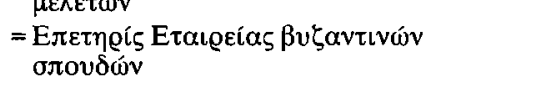 \\
\hline & & $\begin{array}{l}\text { EpetKyklMel } \\
\text { EpigrAnat }\end{array}$ & 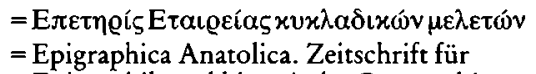 \\
\hline Dacia & $=\mathrm{Dacia}$ & & Epigraphik und historische Geographie \\
\hline Dacoromania & $\begin{aligned}=\text { Dacoromania. Jahrbuch für östliche } \\
\text { Latinität }\end{aligned}$ & Epigraphica & $\begin{aligned} & \text { Anatoliens } \\
= & \text { Epigraphica }\end{aligned}$ \\
\hline $\mathrm{DaM}$ & $=$ Damaszener Mitteilungen & EpistEpetAth & 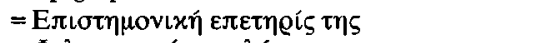 \\
\hline DebrecMuzEvk & = A Debreceni Déri múzeum évkönyve & & 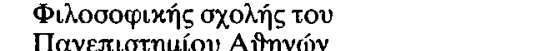 \\
\hline Dédalo & = Dédalo. Revista de arte e arqueologia & EpistEpetPolytThess & 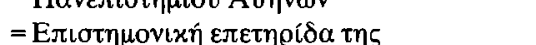 \\
\hline DeltChrA & 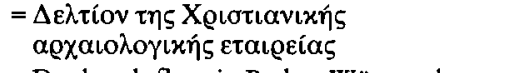 & & 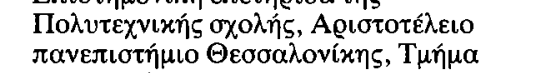 \\
\hline DenkmPflBadWürt & $=$ Denkmalpflege in Baden-Württemberg & & $\alpha \varrho \chi เ \tau \varepsilon \mathcal{H} \tau o ́ v \omega v$ \\
\hline DenkschrWien & $\begin{array}{l}=\text { Österreichische Akademie der } \\
\text { Wissenschaften, Philosophisch- } \\
\text { historische Klasse. Denkschriften }\end{array}$ & EpistEpetThess & 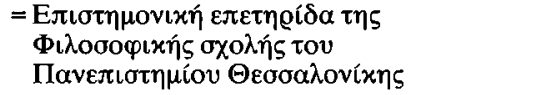 \\
\hline Diadora & $=$ Diadora & Eranos & $=$ Eranos. Acta philologica suecana \\
\hline DialA & $=$ Dialoghi di archeologia & EranosJb & $=$ Eranos-Jahrbuch \\
\hline DialHistAnc & $=$ Dialogues d'histoire ancienne & ErIsr & $=$ Eretz-Israel \\
\hline Dioniso & $=$ Dioniso & EstAAlava & = Estudios de arqueología alavesa \\
\hline DKuDenkmPfl & $=$ Deutsche Kunst und Denkmalpflege & EstMadr & = Estudios de prehistoria y arqueología \\
\hline $\begin{array}{l}\text { DLZ } \\
\text { DocAlb }\end{array}$ & $\begin{array}{l}=\text { Deutsche Literaturzeitung für Kritik der } \\
\text { internationalen Wissenschaft } \\
=\text { Documenta albana }\end{array}$ & EstZaragoza & $\begin{array}{l}\text { = Estudios del Seminario de prehistoria, } \\
\text { arqueología e historia antigua de la } \\
\text { Facultad de filosofía y letras de Zaragoza }\end{array}$ \\
\hline DocALouv & $\begin{aligned} &= \text { Documents d'archéologie régionale. } \\
& \text { Université catholique de Louvain }\end{aligned}$ & $\begin{array}{l}\mathrm{EtACl} \\
\mathrm{EtCl}\end{array}$ & $\begin{array}{l}=\text { Etudes d'archéologie classique } \\
=\text { Les études classique }\end{array}$ \\
\hline DocAMerid & $=$ Documents d'archéologie méridionale & EtClAix & $=$ Etudes classiques . Faculté des lettres et \\
\hline DocEmRomag & $\begin{array}{l}=\text { Documenti. Istituto per } \mathrm{i} \text { beni artistici, } \\
\text { culturali, naturali della regione Emilia- } \\
\text { Romagna }\end{array}$ & Ethnos & $\begin{array}{l}\text { sciences humaines d'Aix } \\
=\text { Ethnos. Revista do Instituto português de } \\
\text { arqueologia, história e etnografia }\end{array}$ \\
\hline Dodone & $=\Delta \omega \delta \dot{\omega} v \eta$. I $\omega \alpha \dot{\alpha} v v v \alpha$ & EtP & $=$ Etudes de papyrologie \\
\hline DOP & $=$ Dumbarton Oaks Papers & EtPézenas & = Etudes sur Pézenas et l'Hérault \\
\hline
\end{tabular}




\begin{tabular}{|c|c|c|c|}
\hline Etruscans & $\begin{array}{l}=\text { Etruscans. Bulletin of the Etruscan } \\
\text { Foundation }\end{array}$ & Geo-archeologia & $\begin{aligned} &= \text { Geo-archeologia. Periodico } \\
& \text { dell'Associazione geo-archeologica } \\
& \text { italiana }\end{aligned}$ \\
\hline EtTrav & $\begin{array}{l}=\text { Etudes et travaux. Studia i prace. Travaux } \\
\text { du Centre d'archéologie méditerranéenne } \\
\text { de l'Académie des sciences polonaise }\end{array}$ & $\begin{array}{l}\text { Gerión } \\
\text { Germania }\end{array}$ & $\begin{aligned} & \text { Italiana } \\
= & \text { Gerión (Madrid) } \\
= & \text { Germania }\end{aligned}$ \\
\hline Eunomia & $\begin{array}{l}=\text { Eunomia. Ephemeridis Listy filologické } \\
\text { supplementum }\end{array}$ & Gesta & $\begin{array}{l}=\text { Gesta. International Center of Medieval } \\
\text { Art }\end{array}$ \\
\hline Euphrosyne & $=$ Euphrosyne. Revista de filologia clássica & GettyMusJ & $=$ The J. Paul Getty Museum Journal \\
\hline Expedition & $=$ Expedition & GGA & $=$ Göttingische Gelehrte Anzeigen \\
\hline ExtremA & = Extremadura arqueológica & $\begin{array}{l}\text { GiornFilFerr } \\
\text { GiornItFil } \\
\text { GiornStorLun } \\
\text { GiRoccPalermo }\end{array}$ & $\begin{array}{l}=\text { Giornale filologico ferrarese } \\
=\text { Giornale italiano di filologia } \\
=\text { Giornale storico della Lunigiana } \\
=\text { Giglio di roccia }\end{array}$ \\
\hline Faenza & $=$ Faenza & GlasAJ & = Glasgow Archaeological Journal \\
\hline Faventia & = Faventia. Facultat de lletres, Barcelona & GlasBeograd & = Glasnik. Srpsko arheološko društvo \\
\hline FBerBadWürt & $\begin{aligned}= & \text { Forschungen und Berichte zur Vor- und } \\
& \text { Frühgeschichte in Baden-Württemberg }\end{aligned}$ & $\begin{array}{l}\text { GlasSarajevo } \\
\text { Glotta }\end{array}$ & $\begin{array}{l}=\text { Glasnik Zemaljskog muzeja u Sarajevu } \\
=\text { Glotta }\end{array}$ \\
\hline FelRav & $=$ Felix Ravenna & Gnomon & $=$ Gnomon \\
\hline Das Fenster & $\begin{array}{l}=\text { Das Fenster in der Halle der } \\
\text { Kreissparkasse Köln }\end{array}$ & GodMuzPlov & $\begin{array}{l}\text { = Godišnik na Narodnija arheologičeski } \\
\text { muzej Plovdiv. Annuaire du Musée } \\
\text { national archéologique Plovdiv }\end{array}$ \\
\hline FichEpigr & $\begin{aligned}= & \text { Ficheiro epigráfico. Suplemento de } \\
& \text { "Conimbriga }\end{aligned}$ & GodZborSkopje & $\begin{aligned}= & \text { Godišen zbornik na Filozofskiot fakultet } \\
& \text { na Universitetot vo Skopje }\end{aligned}$ \\
\hline Figlina & $\begin{aligned}= & \text { Figlina. Documents du Laboratoire de } \\
& \text { céramologie de Lyon }\end{aligned}$ & GorLet & $\begin{aligned}= & \text { Goriški letnik. Zbornik Goriškega } \\
\text { muzeja } & \end{aligned}$ \\
\hline $\begin{array}{l}\text { FoggArtMusAcqu } \\
\text { FolA }\end{array}$ & $=$ Fogg Art Museum. Acquisitions 19xx & GöttMisz & $\begin{array}{l}=\text { Göttinger Miszellen. Beiträge zur } \\
\text { ägyptologischen Diskussion }\end{array}$ \\
\hline FolOr & $=$ Folia orientalia & GraRaspr & $=$ Građa i rasprave. Arheološki muzej Istre, \\
\hline Fonaments & $\begin{aligned}= & \text { Fonaments. Prehistòria i mon antic als } \\
& \text { Paisos Catalans. }\end{aligned}$ & GrazBeitr & $\begin{aligned} & \text { Pula } \\
= & \text { Grazer Beiträge }\end{aligned}$ \\
\hline Fondamenti & $\begin{array}{l}=\text { Fondamenti. Rivista quadrimestrale di } \\
\text { cultura }\end{array}$ & GrLatOr & $\begin{aligned}= & \text { Graecolatina et orientalia. Zborník } \\
& \text { Filozofickej fakulty Univerzity } \\
& \text { Komenského }\end{aligned}$ \\
\hline $\begin{array}{l}\text { FontAPos } \\
\text { Fornyännen }\end{array}$ & $=$ Fontes archaeologici Posnanienses & GrLatPrag & $=$ Graecolatina Pragensia \\
\hline Fornvännen & $\begin{array}{l}=\text { Fornvännen. Tidskrift för svensk } \\
\text { antikvarisk forskning }\end{array}$ & $\begin{array}{l}\text { GrRomByzSt } \\
\text { Gymnasium }\end{array}$ & $\begin{array}{l}=\text { Greek, Roman and Byzantine Studies } \\
=\text { Gymnasium }\end{array}$ \\
\hline Forum & $\begin{array}{l}=\text { Forum. Revue du Groupe d'archéologie } \\
\text { antique }\end{array}$ & & \\
\hline FrühMitAltSt & $\begin{array}{l}\text { = Frühmittelalterliche Studien. Jahrbuch } \\
\text { des Instituts für Frühmittelalterforschung } \\
\text { der Universität Münster }\end{array}$ & Habis & = Habis. Universidad de Sevilla. \\
\hline FuB & $\begin{aligned}= & \text { Forschungen und Berichte. Staatliche } \\
& \text { Museen zu Berlin }\end{aligned}$ & HambBeitrA & $\begin{aligned} & \text { Arqueología, filología clásica } \\
= & \text { Hamburger Beiträge zur Archäologie }\end{aligned}$ \\
\hline FuBerBadWürt & $=$ Fundberichte aus Baden-Württemberg & HambBeitrNum & $=$ Hamburger Beiträge zur Numismatik \\
\hline $\begin{array}{l}\text { FuBerHessen } \\
\text { FuBerÖ }\end{array}$ & $\begin{array}{l}=\text { Fundberichte aus Hessen } \\
=\text { Fundberichte aus Österreich }\end{array}$ & Handlingar & $\begin{array}{l}=\text { Kungl. vitterhets historie och antikvitets } \\
\text { akademiens handlingar. Antikvariska } \\
\text { serien }\end{array}$ \\
\hline $\begin{array}{l}\text { FuBerSchwab } \\
\text { FuF }\end{array}$ & = Fundberichte aus Schwaben & HarvStClPhil & $=$ Harvard Studies in Classical Philology \\
\hline & $=$ Forschungen und Fortschritte & $\begin{array}{l}\text { HarvTheolR } \\
\text { HefteABern }\end{array}$ & $\begin{array}{l}=\text { The Harvard Theological Review } \\
=\text { Hefte des Archäologischen Seminars der } \\
\text { Universität Bern }\end{array}$ \\
\hline GacNum & = Gaceta numismática (Barcelona) & Helike & $\begin{aligned} &= \text { Helike. Universidad nacional de } \\
& \text { educación a distancia, Centro regional de }\end{aligned}$ \\
\hline Gades & $\begin{aligned}= & \text { Gades. Revista del Colegio universitario } \\
& \text { de filosofía y letras }\end{aligned}$ & Helikon & $\begin{aligned} & \text { Elche } \\
= & \text { Helikon }\end{aligned}$ \\
\hline Gallaecia & = Gallaecia (Santiago de Campostela) & Helinium & $=$ Helinium \\
\hline Gallia & $\begin{array}{l}=\text { Gallia. Fouilles et monuments } \\
\text { archéologiques en France métropolitaine. }\end{array}$ & Helios & $\begin{aligned}= & \text { Helios. A journal devoted to critical and } \\
& \text { methodological studies of classical }\end{aligned}$ \\
\hline GalliaInf & $\begin{array}{l}=\text { Gallia informations. Préhistoire et } \\
\text { histoire } \\
=\text { Gallia oréhistoire }\end{array}$ & Hellenikajb & $=\begin{array}{l}\text { Hellenika. Jahrbuch für die Freunde } \\
\text { Griechenlands }\end{array}$ \\
\hline $\begin{array}{l}\text { GalliaPrehist } \\
\text { GaR }\end{array}$ & $=$ Gallia préhistoire & HelvA & $=$ Helvetia archaeologica \\
\hline GazBA & $=$ Gazette des beaux-arts & Hephaistos & $\begin{aligned}= & \text { Hephaistos. Kritische Zeitschrift zur } \\
& \text { Theorie und Praxis der Archäologie und }\end{aligned}$ \\
\hline Genava & $=$ Genava & & angrenzender Wissenschaften \\
\hline
\end{tabular}




Hermes
Hesperia
Hispania
HispAnt
HispAntEpigr
HispEpigr
Historia
Historica
HistriaA
HistSprF
Horos
HuelvaA
Humanitas
HumBild
HZ

Ilerda
Iliria
IllinCISt
ILN
Index
IndiceHistEsp
IndogermF
IndUnArtB
InsFulc
IntJNautA
InvLuc
Ipek
Iran

$\begin{array}{ll}\text { IranAnt } & =\text { Iranica antiqua } \\ \text { Iraq } & =\text { Iraq } \\ \text { IsrExplJ } & =\text { Israel Exploration Journal } \\ \text { IsrMusJ } & =\text { The Israel Museum Journal } \\ \text { IsrMusN } & =\text { The Israel Museum News } \\ \text { IsrNumJ } & =\text { Israel Numismatic Journal } \\ \text { IstanbAMüzYll } & =\text { Istanbul Arkeoloji müzeleri yilliğı } \\ \text { IstMitt } & =\text { Istanbuler Mitteilungen } \\ \text { Italica } & =\text { Italica. Cuadernos de trabajos de la } \\ & \text { Escuela española de historia y arqueología } \\ \text { ItNostr } & \text { en Roma } \\ \text { IzvBurgas } & =\text { Italia nostra } \\ \text { IzvMuzJužBalg } & =\text { Izvestija na Narodnija muzej Burgas } \\ & =\text { Izvestija na muzeite ot Južna Bălgarija. } \\ \text { IzvVarna } & \text { Bulletin des musées de la Bulgarie du Sud }\end{array}$

Jábega
= Hermes. Zeitschrift für klassische Philologie

$=$ Hesperia. Journal of the American School of Classical Studies at Athens

= Hispania. Revista española de historia

= Hispania antiqua. Revista de historia antigua

= Hispania antiqua epigraphica

$=$ Hispania epigraphica

= Historia. Zeitschrift für Alte Geschichte

= Historica. Academia RSR, Centrul de istorie, filologie şi etnografie din Craiova

$=$ Histria archeologica

$=$ Historische Sprachforschung

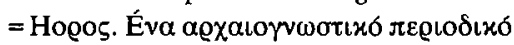

$=$ Huelva arqueológica

$=$ Humanitas

$=$ Humanistische Bildung

$=$ Historische Zeitschrift

$=$ Ilerda. Instituto de estudios ilerdenses (Lérida)

$=$ Iliria

= Illinois Classical Studies

$=$ The Illustrated London News

= Index. Quaderni camerti di studi romanistici

$=$ Indice histórico español

= Indogermanische Forschungen

= Indiana University Art Museum Bulletin

$=$ Insula Fulcheria

= International Journal of Nautical Archaeology

$=$ Invigilata lucernis

$=$ Jahrbuch für prähistorische und ethnographische Kunst

$=$ Iran. Journal of the British Institute of Persian Studies

Iranica antiqua

= Israel Numismatic Journal

= Istanbul Arkeoloji müzeleri yıllığı

Istanbuler Mitteilungen

Escuela española de historia y arqueología en Roma

= Izvestija na Narodnija muzej Burgas

Bulletin des musées de la Bulgarie du Sud

Izvestija na Narodnija muzej Varna

$=$ Jábega. Revista de la Disputación
provincial de Málaga
JadrZbor

Jahresgabe

JARCE

JASc

$\mathrm{JbAChr}$

JbBadWürt

JbBerlMus

JbBernHistMus

JberAugst

JberBasel

JberBayDenkmPfl

JberProVindon

JberVgFrankf

JberZürich

JbGött

JbHambKuSamml

JbKleinasF

JbKuGewHamb

JbKuHistSamml

JbMünch

JbMusLinz

JbÖByz

JbPreussKul

JbSchwUrgesch

JbZMusMainz

JCunSt

JdI

JEA

JEOL

JFieldA

JGS

JHS

JJurP

JMedA

JMedAnthrA

JMithrSt

JNES

JNG

JPrehistRel

JRA
$=$ Jadranski zbornik. Prilozi za povijest

Istre, Rijeke i Hrvatskog primorja

= Winckelmann-Gesellschaft, Stendal

= Journal of the American Research Center in Egypt

= Journal of Archaeological Science

$=$ Jahrbuch für Antike und Christentum

$=$ Jahrbuch der Staatlichen

Kunstsammlungen in Baden-

Württemberg

= Jahrbuch der Berliner Museen

$=$ Jahrbuch des Bernischen Historischen Museums in Bern

$=$ Jahresberichte aus Augst und Kaiseraugst

= Jahresbericht der Archäologischen

Bodenforschung des Kantons Basel-Stadt

$=$ Jahresbericht der Bayerischen

Bodendenkmalpflege

$=$ Jahresbericht. Gesellschaft Pro Vindonissa

= Jahresbericht des Instituts für

Vorgeschichte der Universität Frankfurt

a. $M$.

$=$ Jahresbericht. Schweizerisches

Landesmuseum Zürich

= Jahrbuch der Akademie der

Wissenschaften in Göttingen

= Jahrbuch der Hamburger

Kunstsammlungen

= Jahrbuch für kleinasiatische Forschung

= Jahrbuch des Museums für Kunst und Gewerbe in Hamburg

= Jahrbuch der Kunsthistorischen

Sammlungen in Wien

$=$ Jahrbuch. Bayerische Akademie der

Wissenschaften

= Jahrbuch des Oberösterreichischen

Musealvereins

= Jahrbuch der Österreichischen

Byzantinistik

= Jahrbuch Preussischer Kulturbesitz

= Jahrbuch der Schweizerischen

Gesellschaft für Ur- und Frühgeschichte

$=$ Jahrbuch des Römisch-germanischen

Zentralmuseums Mainz

$=$ Journal of Cuneiform Studies

$=$ Jahrbuch des Deutschen Archäologischen

Instituts

$=$ The Journal of Egyptian Archaeology

= Jaarbericht van het Vooraziatischegyptisch genootschap Ex Oriente Lux

= Journal of Field Archaeology

$=$ Journal of Glass Studies

$=$ The Journal of Hellenic Studies

= Journal of Juristic Papyrology

= Journal of Mediterranean Archaeology

= Journal of Mediterranean Anthropology

and Archaeology

= Journal of Mithraic Studies

= Journal of Near Eastern Studies

= Jahrbuch für Numismatik und Geldgeschichte

$=$ Journal of Prehistoric Religion

= Journal of Roman Archaeology 
JRomMilSt

JRomPotSt

JRS

JSav

JSchrVgHalle

JSemSt

Jura

JWaltersArtGal

JWCI
= Journal of Roman Military Equipment Studies

= Journal of Roman Pottery Studies

$=$ The Journal of Roman Studies

$=$ Journal des savants

= Jahresschrift für mitteldeutsche

Vorgeschichte

$=$ Journal of Semitic Studies

$=$ Jura. Rivista internazionale di diritto romano e antico.

$=$ The Journal of the Walters Art Gallery

= Journal of the Warburg and Courtaud Institutes

\section{Kadmos \\ Kairos \\ Karthago \\ Kêmi}

KentAR

Kernos

Klearchos

Klio

Kobie

Koinonia

Kokalos

KölnJbVFrühGesch

KraSoob

KraSoobInstA

Kratylos

\section{KretChron}

Ktema

KuGeschAnz

Kuml

Kunstchronik

$\mathrm{KuOr}$

Kush

KuWeltBerlMus KypSpud

Labeo

Lampas

LandKunVierJBl

LangOrAnc

Latinitas

$=$ Kadmos

= Kairos. Zeitschrift für Judaistik und Religionswissenschaft

= Karthago. Revue d'archéologie africaine

$=$ Kêmi. Revue de philologie et

d'archéologie égytiennes et coptes

$=$ Kent Archaeological Review

$=$ Kernos. Revue internationale et pluridisciplinaire de religion grecque antique

$=$ Klearchos

$=$ Klio

= Kobie. Grupo espeleológico vizcaíno (Bilbao)

$=$ Koinonia

$=\mathrm{K} \Omega \mathrm{KA} \Lambda \mathrm{O} \Sigma$. Studi pubblicati di Palermo

$=$ Kölner Jahrbuch für Vor- und

Frühgeschichte

$=$ Kratkie soobščenija o dokladach i polevych issledovanijach Instituta archeologii

$=$ Kratylos. Kritisches Berichts- und Rezensionsorgan für indogermanische und allgemeine Sprachwissenschaft

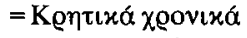

= Ktema. Civilisations de l'Orient, de la

Grèce et de Rome antique

$=$ Kunstgeschichtliche Anzeigen

$=\mathrm{Kuml}$

$=$ Kunstchronik

$=$ Kunst des Orients

= Kush. Journal of the Sudan Antiquities Service

$=$ Kunst der Welt in den Berliner Museen

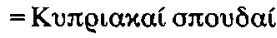

= Labeo. Rassegna di diritto romano

= Lampas. Tijdschrift voor nederlandse classici et linguistique.

$\begin{array}{ll}\text { Latomus } & \text { Latomus. Revue d'études latines } \\ \text { Levant } & \text { Levant. Journal of the British School of } \\ & \text { Archaeology in Jerusalem and the British } \\ & \text { Institute at Amman for Archaeology and } \\ & \text { History } \\ = & \text { Listy filologické } \\ = & \text { Libyan Studies } \\ = & \text { Libya antiqua } \\ \text { LF } & \text { Libyca. Bulletin du Service des antiquités. } \\ \text { LibSt } & \text { Archéologie, épigraphie } \\ \text { LibyaAnt } & \text { Libyca. Travaux du Laboratoire } \\ \text { LibycaBServAnt } & \text { d'anthropologie et d'archéologie } \\ \text { LibycaTrav } & \text { préhistorique du Musée du Bardo } \\ = & \text { Linguistica, epigrafia, filologia italica } \\ = & \text { Lucentum. Anales de la Universidad de } \\ \text { LingIt } & \text { Alicante. Prehistoria, arqueología e } \\ \text { Lucentum } & \text { historia antigua } \\ = & \text { Lustrum }\end{array}$
dall'Istituto di storia antica dell'Università

$=$ Kratkie soobščenija Instituta archeologii

$=$ Landeskundliche Vierteljahrsblätter. Trier

$=$ Langues orientales anciennes. Philologie

MacActaA
MAGesGraz
Maia
Mainake
MAInstUngAk

= Macedoniae acta archaeologica

= Mitteilungen der Archäologischen Gesellschaft Graz

= Maia

= Mainake

$=$ Mitteilungen des Archäologischen Instituts der Ungarischen Akademie der Wissenschaften

MainzZ

Makedonika

MAnthrWien

MarbWPr

Mari

Marisia

Marsyas

MascaJ

MascaP

MatABelorSSR

MatASevPrič

MatCercA

MatIsslA

MatStar

MatTestiCl

MatWczes

MBIVFrühGesch

MDAVerb

MDIK

$=$ Mainzer Zeitschrift

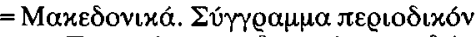

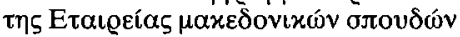

$=$ Mitteilungen der Anthropologischen Gesellschaft in Wien

= Marburger Winckelmann-Programm

$=$ Mari. Annales de recherches interdisciplinaires

= Marisia. Studii şi materiale. Arheologie, istorie, etnografie

= Marsyas. Studies in the History of Art

= Masca Journal. Museum Applied Science Center for Archaeology, University of Pennsylvania

= Masca Research Papers in Science and Archaeology

= Materialy po archeologii BSSR

= Materialy po archeologii severnogo Pričernomor'ja

$=$ Materiale şi cercetări arheologice

= Materialy $\mathrm{i}$ issledovanija po archeologii SSSR

= Materiały starożytne

= Materiali e discussioni per l'analisi dei testi classici

= Materiały wczesnośredniowieczne

$=$ Mitteilungsblatt für Vor- und Frühgeschichte

$=$ Mitteilungen des Deutschen Archäologen-Verbandes e. V.

$=$ Mitteilungen des Deutschen Archäologischen Instituts, Abteilung Kairo 
MDOG

Meander

MedA

MeddelGlypt

MeddelLund

MeddelThor

MededRom

MedelhavsMusB

MedHistR

MedievA

MEFRA

MelBeyrouth

MelCasaVelazquez

MemAcInscr

MemAmAc

MemAnt

MemAntFr

MemBarcelA

MemBologna

MemHistAnt

MemInstNatFr

MemLinc

MemMusAProvinc MemNap

MemPontAc

MemStorFriuli

Mesopotamia

Messana

Métis

MetrMusJ

Minia

Minos

MInstWasser

MIO

MiscAMataro

MiscCrAnt
$=$ Mitteilungen der Deutschen OrientGesellschaft zu Berlin

= Meander. Miesięcznik poświęcony kulturze świata starożytnego

= Mediterranean Archaeology

= Meddelelser fra Ny Carlsberg Glyptotek København

= Meddelanden fran Lunds Universtitets Historiska Museum

= Meddellelser fra Thorwaldsens Museum

$=$ Mededelingen van het Nederlands Instituut te Rome. Antiquity

= Medelhavsmuseet. Bulletin (Stockholm)

$=$ Mediterranean Historical Review

= Medieval Archaeology. Journal of the Society for Medieval Archaeology

= Mélanges de l'Ecole française de Rome. Antiquité

= Mélanges de l'Université Saint-Joseph

= Mélanges de la Casa de Velázquez.

Antiquité et moyen âge

= Mémoires de l'Académie des inscriptions et belles-lettres

$=$ Memoirs of the American Academy in Rome

$=$ Memoria antiquitatis. Acta Musei

Petrodavensis. Revista Muzeului arheologic Piatra Neamł

$=$ Mémoires de la Société nationale des antiquitaires de France

$=$ Memoria. Universidad de Barcelona Instituto de arqueología y prehistoria.

$=$ Atti de la Accademia delle scienze dell'Istituto di Bologna. Memorie

$=$ Memorias de historia antigua. Universidad de Oviedo

= Mémoires de l'Institut national de France

= Memorie. Atti della Accademia nazionale dei Lincei, Classe di scienze morali, storiche e filologiche

$=$ Memorias de los museos arqueologícos

= Memorie dell'Accademia di archeologia, lettere e belle arti di Napoli

$=$ Memorie. Atti della Ponteficia accademia romana di archeologia

$=$ Memorie storiche forogiuliesi

= Mesopotamia. Rivista di archeologia

= Messana. Rassegna di studi filologici, linguistici e storici

= Métis. Revue d'anthropologie du monde grec ancien

- Metropolitan Museum Journal

$=$ Minia (Braga)

$=$ Minos

$=$ Mitteilungen. Leichtweiss-Institut für Wasserbau der Technischen Universität Braunschweig.

$=$ Mitteilungen des Instituts für Orientforschung

= Miscellánies arqueològiques sobre Mataró i el Maresme

= Miscellanea di studi di letteratura cristiana antica

\begin{tabular}{|c|c|}
\hline MKuHistFlorenz & $\begin{array}{l}\text { = Mitteilungen des Kunsthistorischen } \\
\text { Instituts Florenz }\end{array}$ \\
\hline MKul & $=$ Mitteilungen zur Kulturkunde \\
\hline MM & = Madrider Mitteilungen \\
\hline Mnemosyne & $=$ Mnemosyne \\
\hline MonAnt & $=$ Monumenti antichi \\
\hline MonPiot & $\begin{aligned}= & \text { Monuments et mémoires. Fondation } \mathrm{E} . \\
& \text { Piot }\end{aligned}$ \\
\hline Monumentet & = Monumentet \\
\hline MÖNumGes & $\begin{array}{l}=\text { Mitteilungen der Österreichischen } \\
\text { Numismatischen Gesellschaft }\end{array}$ \\
\hline MPrähistKomWien & $\begin{aligned}= & \text { Mitteilungen der Prähistorischen } \\
& \text { Kommission der Österreichischen } \\
& \text { Akademie der Wissenschaften }\end{aligned}$ \\
\hline MSchwUrFrühGesch & $\begin{aligned}= & \text { Mitteilungsblatt der Schweizerischen } \\
& \text { Gesellschaft für Ur- und Frühgeschichte }\end{aligned}$ \\
\hline MüJb & $=$ Münchner Jahrbuch der bildenden Kunst \\
\hline MünchStSprWiss & $\begin{aligned}= & \text { Münchener Studien zur } \\
& \text { Sprachwissenschaft }\end{aligned}$ \\
\hline MünstBeitr & $\begin{aligned}= & \text { Münstersche Beiträge zur antiken } \\
& \text { Handelsgeschichte }\end{aligned}$ \\
\hline Murcia & $\begin{array}{l}=\text { Murcia. Boletín informativo de la Excma } \\
\text { Diputación provincial }\end{array}$ \\
\hline MusAfr & $\begin{array}{l}=\text { Museum Africum. West African Journal } \\
\text { of Classical and Related Studies }\end{array}$ \\
\hline MusCrit & $=$ Museum criticum \\
\hline Muse & $\begin{array}{l}=\text { Muse. Annual of the Museum of Art and } \\
\text { Archaeology, University of Missouri, } \\
\text { Columbia }\end{array}$ \\
\hline Muséon & $=$ Le muséon. Revue d'études orientales \\
\hline Museum & $\begin{aligned} &= \text { Museum. A quarterly review published } \\
& \text { by UNESCO }\end{aligned}$ \\
\hline MusGalit & $=$ Musei e gallerie d'Italia \\
\hline MusHaaretz & $=$ Museum Haaretz, Tel Aviv. Yearbook \\
\hline MusHelv & $=$ Museum helveticum \\
\hline MusKöln & $=$ Museen in Köln. Bulletin \\
\hline MusNotAmNumSoc & $\begin{aligned} &= \text { Museum Notes. The American } \\
& \text { Numismatic Society }\end{aligned}$ \\
\hline MusPontevedr & $=$ El Museo de Pontevedra \\
\hline MusTusc & $=$ Museum Tusculanum. København \\
\hline MuzEvkSzeged & = A Móra Ferenc múzeum évkönyve \\
\hline MuzNaf & $=$ Muzeul naţional. Bucareşti \\
\hline MuzPamKul & $=$ Muzei i pametnici na kulturata \\
\hline
\end{tabular}

NachrAkGött

= Nachrichten der Akademie der Wissenschaften in Göttingen

NapNobil

NassAnn

NBWorcArtMus

NEphemSemEpigr

Nestor

NewsletterPotTech

Nikephoros

NomChron

Norba

NotAHisp
$=$ Napoli nobilissima

= Nassauische Annalen

$=$ News Bulletin and Calendar. Worcester Art Museum

$=$ Neue Ephemeris für semitische Epigraphik

$=$ Nestor

$=$ Newsletter. Department of pottery technology, University of Leiden

$=$ Nikephoros. Zeitschrift für Sport und Kultur im Altertum

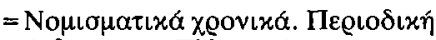

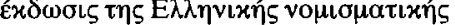

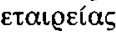

= Norba. Revista de arte, geografía e historia (Cáceres)

= Noticiario arqueológico hispánico. Arqueología 


\begin{tabular}{|c|c|c|c|}
\hline NotAHispPrehistoria & $\begin{array}{l}=\text { Noticiario arqueológico hispánico. } \\
\text { Prehistoria }\end{array}$ & OrpheusThracSt & $\begin{array}{l}\text { = Orpheus. Journal of Indo-European, } \\
\text { Paleao-Balkan and Thracian studies }\end{array}$ \\
\hline NotAllumiere & $\begin{aligned}= & \text { Notiziario. Museo civico, Associazione } \\
& \text { archeologica, Allumiere }\end{aligned}$ & OsjZbor & $=$ Osječki zbornik \\
\hline NotALomb & $\begin{aligned} & \text { archeologica, Allumiere } \\
&= \text { Notiziario. Soprintendenza archeologica } \\
& \text { della Lombardia }\end{aligned}$ & OstbGrenzm & $\begin{array}{l}\text { = Ostbairische Grenzmarken. Passauer } \\
\text { Jahrbuch für Geschichte, Kunst und } \\
\text { Volkskunde }\end{array}$ \\
\hline \multirow[t]{2}{*}{ NotMilano } & \multirow{3}{*}{$\begin{array}{l}=\text { Notizie del Chiostro del Monastero } \\
\text { maggiore. Rassegna di studi del Civico } \\
\text { museo archeologico e del Civico gabinetto } \\
\text { numismatico di Milano }\end{array}$} & OudhMeded & $\begin{array}{l}=\text { Oudheidkundige mededelingen uit het } \\
\text { Rijksmuseum van oudheden te Leiden }\end{array}$ \\
\hline & & OxfJA & $=$ Oxford Journal of Archaeology \\
\hline NouvClio & & \multirow[t]{2}{*}{ OxfStPhilos } & \multirow[t]{2}{*}{$=$ Oxford Studies in Ancient Philosophy } \\
\hline Novaensia & $\begin{aligned}= & \text { Novaensia. Badania Ekspedycji } \\
& \text { archeolgicznej Uniwersytetu } \\
& \text { warszawskiego w Novae }\end{aligned}$ & & \\
\hline \multirow{4}{*}{$\begin{array}{l}\text { NSc } \\
\text { NubChr } \\
\text { NubLet } \\
\text { NumAntCl }\end{array}$} & $=$ Notizie degli scavi di antichità & \multirow[t]{3}{*}{ Pact } & \multirow{3}{*}{$\begin{array}{l}=\text { Pact. Revue du Groupe européen d'études } \\
\text { pour les techniques physiques, chimiques } \\
\text { et mathématiques appliquées à } \\
\text { l'archéologie }\end{array}$} \\
\hline & $=$ Nubia christiana & & \\
\hline & $=$ Nubian Letters & & \\
\hline & $\begin{aligned}= & \text { Numismatica e antichità classiche. } \\
& \text { Quaderni ticinesi }\end{aligned}$ & \multirow[t]{2}{*}{ Padusa } & \multirow{2}{*}{$\begin{array}{l}=\text { Padusa. Bollettino del Centro polesano di } \\
\text { studi storici, archeologici ed etnografici, } \\
\text { Rovigo }\end{array}$} \\
\hline Numantia & $\begin{aligned}= & \text { Numantia. Investigaciones arqueológicas } \\
& \text { en Castilla y León }\end{aligned}$ & & \\
\hline NumChron & $=$ The Numismatic Chronicle & Paideuma & = Paideuma. Mitteilungen zur Kulturkunde \\
\hline Numen & $=$ Numen & Palaeohistoria & $=$ Palaeohistoria \\
\hline NumEpigr & $=$ Numizmatika i epigrafika & Palladio & $=$ Palladio. Rivista di storia dell'architettura \\
\hline Numisma & $\begin{aligned}= & \text { Numisma. Revista de la Sociedad } \\
& \text { iberoamericana de estudios numismáticos }\end{aligned}$ & Pallas & $\begin{aligned}= & \text { Pallas. Annales publiées par la Faculté des } \\
& \text { lettres et sciences humaines de Toulouse }\end{aligned}$ \\
\hline NumismaticaRom & $=$ Numismatica & PamA & = Památky archeologické \\
\hline Numizmatičar & $=$ Numizmatičar & Pan & $=$ Pan . Studi dell'Istituto di filologia latina \\
\hline \multirow[t]{2}{*}{ Nummus } & \multirow{2}{*}{$\begin{array}{l}=\text { Nummus. Sociedade portuguesa de } \\
\text { numismatica }\end{array}$} & Pantheon & $=$ Pantheon \\
\hline & & Partenope & $=$ Partenope \\
\hline NumZ & $=$ Numismatische Zeitschrift & PEQ & = Palestine Exploration Quarterly \\
\hline \multirow[t]{3}{*}{ NuovDidask } & \multirow[t]{2}{*}{$=$ Nuovo Didaskaleion } & Peristil & $\begin{aligned}= & \text { Peristil. Zbornik radova za povijest } \\
& \text { umjetnosti }\end{aligned}$ \\
\hline & & Persica & $=$ Persica \\
\hline & $=$ Oriens antiquus & Peuce & $\begin{aligned}= & \text { Peuce. Studii şi comunicări de istorie şi } \\
& \text { arheologie }\end{aligned}$ \\
\hline OccasPublClSt & $\begin{array}{l}=\text { Occasional Publications in Classical } \\
\text { Studies }\end{array}$ & Philologus & $\begin{aligned}= & \text { Philologus. Zeitschrift für das klassische } \\
& \text { Altertum }\end{aligned}$ \\
\hline \multirow{2}{*}{$\begin{array}{l}\text { Offa } \\
\text { Ogam } \\
\text { Oikumene }\end{array}$} & \multirow{2}{*}{$\begin{aligned}= & \text { Offa } \\
= & \text { Ogam } \\
= & \text { Oikumene. Studia ad historiam antiquam } \\
& \text { classicam et orientalem pertinentia }\end{aligned}$} & \multirow{2}{*}{$\begin{array}{l}\text { Phoenix } \\
\text { PhoenixExOrLux }\end{array}$} & \multirow{3}{*}{$\begin{array}{l}=\text { Phoenix. The Classical Association of } \\
\text { Canada } \\
=\text { Phoenix. Bulletin uitgegeven door het } \\
\text { Vooraziatisch-Egyptisch Genootschap Ex } \\
\text { Oriente Lux }\end{array}$} \\
\hline & & & \\
\hline ÖJh & \multirow{4}{*}{$\begin{aligned} &= \text { Jahreshefte des Österreichischen } \\
& \text { Archäologischen Institutes in Wien } \\
&= \text { Orientalistische Literaturzeitung } \\
&= \text { Opuscula Atheniensia } \\
& \text { = Opuscula Romana } \\
&=\text { Opus. Rivista internazionale per la storia } \\
& \text { economica e sociale dell'antichità }\end{aligned}$} & Phoibos & \\
\hline OLZ & & Phronesis & $=$ Phronesis \\
\hline OpAth & & Picus & $\begin{aligned}= & \text { Picus. Studi e ricerche sulle Marche } \\
& \text { nell'antichità }\end{aligned}$ \\
\hline $\begin{array}{l}\text { OpRom } \\
\text { Opus }\end{array}$ & & Platon & 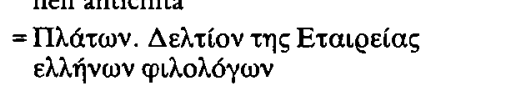 \\
\hline OpuscFin & $=$ Opuscula Instituti Romani Finlandiae & Poetica & $=$ Poetica \\
\hline OrChr & $=$ Oriens christianus & Polemon & 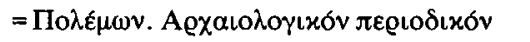 \\
\hline OrChrPer & $=$ Orientalia christiana periodica & PompHercStab & $=$ Pompeii, Herculaneum, Stabiae. \\
\hline Oretania & = Oretania. Revista de historia, arte, & & $\begin{array}{l}\text { Bollettino. Associazione internazionale } \\
\text { Amici di Pompei }\end{array}$ \\
\hline & $\begin{array}{l}\text { arqueologia. Museo arqueologico de } \\
\text { Linares }\end{array}$ & Pontica & $=$ Pontica \\
\hline Orient & $=$ Orient. The Reports of the Society for & Portugalia & $=$ Portugalia \\
\hline & Near Eastern Studies in Japan & PP & = La parola del passato \\
\hline Orientalia & $=$ Orientalia. Pontificium institutum & PraceA & $=$ Prace archeologiczne \\
\hline & biblicum & PraceMatKodz & $=$ Prace $\mathrm{i}$ materialy Muzeum \\
\hline Origini & $\begin{array}{l}=\text { Origini. Preistoria e protostoria delle } \\
\text { civiltà antiche }\end{array}$ & & archeologicznegoietnograficznegc \\
\hline Orpheus & $\begin{array}{l}\text { civiltà antiche } \\
=\text { Orpheus. Rivista di umanità classica e } \\
\text { cristiana }\end{array}$ & Prakt & 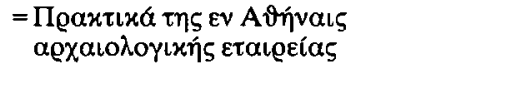 \\
\hline
\end{tabular}

XX 


PraktAkAth
Préhistoire
PreistAlp
PriloziZagreb
PrincViana
ProcAfrClAss
ProcCambrPhilSo
ProcPrehistSoc
Prometheus
ProspAQuad
Prospettiva
Prospezioni
ProvHist
ProvLucca
Pulpudeva
Puteoli
Pyrenae
PZ

\begin{tabular}{|c|c|}
\hline QuadABarcel & $\begin{aligned}= & \text { Quaderns d'arqueologia i història de la } \\
& \text { ciutat (Barcelona) }\end{aligned}$ \\
\hline QuadACagl & $\begin{aligned}= & \text { Quaderni. Soprintendenza archeologica } \\
& \text { per le provincie di Cagliari e Oristano }\end{aligned}$ \\
\hline QuadACal & $\begin{array}{l}=\text { Quaderni del Dipartimento delle arti, } \\
\text { Università della Calabria }\end{array}$ \\
\hline QuadALibia & $=$ Quaderni di archeologia della Libia \\
\hline QuadAPiem & $\begin{array}{l}=\text { Quaderni della Soprintendenza } \\
\text { archeologica del Piemonte }\end{array}$ \\
\hline QuadAReggio & $=$ Quaderni d'archeologia reggiana \\
\hline QuadAVen & $=$ Quaderni di archeologia del Veneto \\
\hline QuadCat & $\begin{aligned}= & \text { Quaderni catanesi di studi classici e } \\
& \text { medievali }\end{aligned}$ \\
\hline QuadChieti & $\begin{array}{l}=\text { Quaderni dell'Istituto di archeologia e } \\
\text { storia antica, Università di Chieti }\end{array}$ \\
\hline QuadErb & $\begin{aligned}= & \text { Quaderni erbesi. Civico museo } \\
& \text { archeologico di Erba }\end{aligned}$ \\
\hline QuadEstMediev & $=$ Quaderns d'estudis medievals \\
\hline QuadIstFilGr & $=$ Quaderni dell'Istituto di filologia greca \\
\hline QuadIstLat & $\begin{aligned}= & \text { Quaderni dell'Istituto di lingua e } \\
& \text { letteratura latina, Università di Roma }\end{aligned}$ \\
\hline QuadLecce & $\begin{array}{l}\text { = Quaderni. Istituto di lingue e letterature } \\
\text { classiche, Facoltà di magistero, Universtà } \\
\text { degli studi, Lecce }\end{array}$ \\
\hline QuadMess & $\begin{array}{l}=\text { Quaderni dell'Istituto di archeologia della } \\
\text { Facoltà di lettere e filosofia della } \\
\text { Università di Messina. }\end{array}$ \\
\hline QuadMusPontecorvo & = Museo civico Pontecorvo. Quaderni \\
\hline QuadProtost & $=$ Quaderni di protostoria \\
\hline QuadStLun & $=$ Quaderni. Centro studi lunensi \\
\hline QuadStor & $=$ Quaderni di storia \\
\hline QuadUrbin & = Quaderni urbinati di cultura classica \\
\hline
\end{tabular}

$=$ Préhistoire

= Preistoria alpina. Museo tridentino di scienze naturali

= Prilozi. Centar za povijesne znanosti

Sveučilišta u Zagrebu, Odjel za arheologiju

= Príncipe di Viana

$=$ The Proceedings of the African Classical Associations

$=$ Proceedings of the Cambridge

Philological Society

$=$ Proceedings of the Prehistoric Society

$=$ Prometheus. Rivista quadrimestrale di studi classici

= Prospezioni archeologiche. Quaderni

= Prospettiva. Rivista di storia dell'arte antica e moderna

$=$ Prospezioni. Bollettino di informazioni scientifiche

$=$ Provence historique

$=\mathrm{La}$ Provincia di Lucca

= Pulpudeva. Semaines philippopolitaines de l'histoire et de la culture thrace

$=$ Puteoli. Studi di storia antica

= Pyrenae. Crónica arqueológica

$=$ Praehistorische Zeitschrift

\author{
Quaternaria
}

$=$ Quaternaria

RA

RAArtLouv

RACFr

RAComo

$\mathrm{RACr}$

RadAkZadar

Radiocarbon

RAE

Raggi

RAlicante

RANarb

RArchBiblMus

RArcheom

RArtMus

RassAPiomb

RassLazio

RassStorSalern

RAssyr

Ratiariensia

Raydan

RBelgNum

RBelgPhilHist

$\mathrm{RBi}$

RCulCiMedioev

RdA

RDAC

RDroitsAnt

REA

REByz

RecConstantine

RechACrac

RechAlb

RedenGedenk

REG

$\mathrm{REg}$

ReiCretActa

REL

Rema
$=$ Revue archéologique

= Revue des archéologues et historiens d'art de Louvain

= Revue archéologique du Centre de la France

= Rivista archeologica dell'antica provincia e diocesi di Como

= Rivista di archeologia cristiana

= Radovi Instituta Jugoslavenske akademije znanosti i umjetnosti u Zadru

$=$ Radiocarbon

$=$ Revue archéologiques de l'Est et du Centre-Est consacrée aux antiquités nationales

$=$ Raggi. Zeitschrift für Kunstgeschichte und Archäologie

$=$ Revista. Instituto de estudios alicantinos

= Revue archéologique de Narbonnaise

= Revista de archivos, bibliotecas y museos

$=$ Revue d'archéométrie

$=\mathrm{L}$ a revue des arts. Musées de France

= Rassegna di archeologia. Associazione archeologica piombinese

$=$ Rassegna del Lazio

$=$ Rassegna storica salernitana

= Revue d'assyriologie et d'archéologie orientale

$=$ Ratiariensia. Studi e materiali mesici e danubiani

$=$ Raydan. Journal of the ancient Yemeni antiquities and epigraphy

$=$ Revue belge de numismatique et de sigillographie

$=$ Revue belge de philologie et d'histoire

$=$ Revue biblique

$=$ Rivista di cultura classica e medioevale

$=$ Rivista di archeologia

= Report of the Department of Antiquities, Cyprus

$=$ Revue internationale des droits de l'antiquité

$=$ Revue des études anciennes

= Revue des études byzantines

= Recueil des notices et mémoires de la Société archéologique du département de Constantine

$=$ Recherches archéologiques. Institut d'archéologie de l'Université de Cracovie

$=$ Recherches albanologiques

= Reden und Gedenkworte. Orden Pour le mérite für Wissenschaften und Künste

$=$ Revue des études grecques

$=$ Revue d'égyptologie

$=$ Rei cretariae romanae fautorum acta

$=$ Revue des études latines

$=$ P $\mu \alpha$ 


\begin{tabular}{|c|c|c|c|}
\hline RendBologna & $\begin{aligned} &= \text { Atti della Accademia delle scienze } \\
& \text { dell'Istituto di Bologna. Rendiconti }\end{aligned}$ & $\begin{array}{l}\mathrm{RStO} \\
\text { RStorAnt }\end{array}$ & $\begin{array}{l}=\text { Rivista degli studi orientali } \\
=\text { Rivista storica dell'antichità }\end{array}$ \\
\hline RendIstLomb & $\begin{aligned}= & \text { Rendiconti. Istituto lombardo, } \\
& \text { Accademia di scienze e lettere }\end{aligned}$ & RStorCal & $=$ Rivista storica calabrese \\
\hline RendLinc & $\begin{aligned}= & \text { Atti dell'Accademia nazionale dei Lincei. } \\
& \text { Classe di scienze morali, storiche e } \\
& \text { filologiche. Rendiconti }\end{aligned}$ & $\begin{array}{l}\text { RStPomp } \\
\text { Rudiae }\end{array}$ & $\begin{array}{l}=\text { Rivista di studi pompeiani } \\
=\text { Rudiae. Ricerche sul mondo classico }\end{array}$ \\
\hline RendNap & $\begin{aligned}= & \text { Rendiconti della Accademia di } \\
& \text { archeologia, lettere e belle arti, Napoli }\end{aligned}$ & & \\
\hline RendPontAc & $\begin{aligned} &= \text { Rendiconti. Atti della Pontificia } \\
& \text { accademia romana di archeologia }\end{aligned}$ & SaalbJb & $\begin{aligned}= & \text { Saalburg-Jahrbuch. Bericht des Saalburg- } \\
& \text { Museums }\end{aligned}$ \\
\hline RepMalta & $\begin{array}{l}=\text { Report on the Working of the Museum } \\
\text { Department for the Year 19xx. Malta, } \\
\text { Department of information }\end{array}$ & Saeculum & $\begin{array}{l}=\text { Saeculum. Jahrbuch für } \\
\text { Universalgeschichte }\end{array}$ \\
\hline Reppal & $=$ Reppal. Centre d'études phéniciennes- & SaggiFen & $=$ Saggi fenici \\
\hline RepSocLibSt & $=$ Annual Report. The Society for Libyan & Saguntum & $\begin{aligned} &= \text { Saguntum. Papeles del Laboratorio de } \\
& \text { arqueología de Valencia }\end{aligned}$ \\
\hline REtArm & $\begin{array}{l}\text { Studies } \\
=\text { Revue des études arméniennes }\end{array}$ & Saitabi & $\begin{aligned}= & \text { Saitabi. Noticiario de historia, arte y } \\
& \text { arqueología de Levante }\end{aligned}$ \\
\hline & $=$ Rivista di filologia e d'istruzione classica & Sandalion & $\begin{aligned}= & \text { Sandalion. Quaderni di cultura classica, } \\
& \text { cristiana e medievale }\end{aligned}$ \\
\hline RGeorgCauc & $\begin{array}{l}=\text { Revue des études géorgiennes et } \\
\text { caucasiennes }\end{array}$ & Sargetia & $=$ Sargetia. Acta Musei (regionalis) devensis \\
\hline $\begin{array}{l}\text { RGirona } \\
\text { RGuimar }\end{array}$ & $\begin{array}{l}=\text { Revista de Girona } \\
=\text { Revista de Guimarães }\end{array}$ & Savaria & $\begin{array}{l}=\text { Savaria. Bulletin der Museen des Komitats } \\
\text { Vas }\end{array}$ \\
\hline RHA & $=$ Revue hittite et asianique & SBBerlin & $=$ Sitzungsberichte der Deutschen \\
\hline RheinMusBonn & $=$ Das Rheinische Landesmuseum Bonn & & Klasse für Sprache, Literatur und Kunst \\
\hline $\begin{array}{l}\text { RHistRel } \\
\text { RhM }\end{array}$ & $\begin{array}{l}\text { = Revue de l'histoire des religions } \\
=\text { Rheinisches Museum für Philologie }\end{array}$ & SBLeipzig & $\begin{aligned}= & \text { Sitzungsberichte der Sächsischen } \\
& \text { Akademie der Wissenschaften zu Leipzig }\end{aligned}$ \\
\hline RicStBrindisi & $\begin{aligned}= & \text { Rivista dell'Istituto nazionale } \\
& \text { d'archeologia e storia dell'arte } \\
= & \text { Ricerche e studi. Museo Francesco }\end{aligned}$ & SBMünchen & $\begin{array}{l}\text { = Bayerische Akademie der Wissenschaften. } \\
\text { Philosophisch-historische Klasse. } \\
\text { Sitzungsberichte }\end{array}$ \\
\hline $\begin{array}{l}\text { RIngIntem } \\
\text { RItNum }\end{array}$ & $\begin{aligned} & \text { Ribezzo, Brindisi } \\
= & \text { Rivista Ingauna e Intemelia } \\
= & \text { Rivista italiana di numismatica e scienze }\end{aligned}$ & SborBrno & $\begin{array}{l}=\text { Sborník prací Filosofické fakulty } \\
\text { Brněnské university. Rada archeologicko- } \\
\text { klasická (E) }\end{array}$ \\
\hline RLouvre & $\begin{aligned} & \text { affini } \\
= & \text { La revue du Louvre et des musées de }\end{aligned}$ & SBWien & $\begin{aligned}= & \text { Sitzungsberichte. } \text { Osterreichische } \\
& \text { Akademie der Wissenschaften }\end{aligned}$ \\
\hline RM & $\begin{aligned} & \text { France } \\
= & \text { Mitteilungen des Deutschen }\end{aligned}$ & ScAnt & $\begin{aligned}= & \text { Scienze dell'antichità. Storia, archeologia, } \\
& \text { antropologia }\end{aligned}$ \\
\hline RNum & $\begin{aligned} & \text { Archäologischen Instituts, Römische } \\
& \text { Abteilung } \\
&=\text { Revue numismatique }\end{aligned}$ & SchildStei & $\begin{aligned} &= \text { Schild von Steier. Beiträge zur Steirischen } \\
& \text { Vor- und Frühgeschichte und } \\
& \text { Münzkunde }\end{aligned}$ \\
\hline RoczMuzWarsz & $=$ Rocznik Muzeum narodowego $\mathrm{w}$ & SchwMüBl & $=$ Schweizer Münzblätter \\
\hline & Warszawie & SchwNumRu & $=$ Schweizerische numimatische Rundschau \\
\hline Romanobarbarica & $=$ Romanobarbarica. Contributi allo studio & ScrCiv & $=$ Scrittura e civiltà \\
\hline RomGens & $\begin{aligned} & \text { mondo barbarico } \\
&= \text { Romana gens. Bollettino } \\
& \text { dell'Associazione archeologica romana }\end{aligned}$ & ScrClIsr & $\begin{aligned} &= \text { Scripta classica Israelica. Yearbook of the } \\
& \text { Israel Society for the Promotion of } \\
& \text { Classical Studies }\end{aligned}$ \\
\hline RömÖ & $=$ Römisches Österreich & ScrHieros & $\begin{aligned}= & \text { Scripta Hierosolymitana. Publications of } \\
& \text { the Hebrew University, Jerusalem }\end{aligned}$ \\
\hline RömQSchr & $\begin{array}{l}\text { = Römische Quartalschrift für christliche } \\
\text { Altertumskunde und Kirchengeschichte }\end{array}$ & ScrMed & $\begin{aligned}= & \text { Scripta Mediterranea. Bulletin of the } \\
& \text { Society for Mediterranean Studies }\end{aligned}$ \\
\hline RPhil & $\begin{array}{l}=\text { Revue de philologie, de littérature et } \\
\text { d'histoire anciennes }\end{array}$ & SetubalA & = Setúbal arqueológica \\
\hline RPorto & $\begin{array}{l}=\text { Revista da Facultade de letras. Serie de } \\
\text { historia. Universidade do Porto }\end{array}$ & $\begin{array}{l}\text { Sibrium } \\
\text { SicA }\end{array}$ & $\begin{array}{l}=\text { Sibrium } \\
=\text { Sicilia archeologica }\end{array}$ \\
\hline RSaintonge & $=$ Revue de la Saintonge et de l'Aunis & SicGymn & $=$ Siculorum gymnasium \\
\hline RScPreist & $=$ Rivista di scienze preistoriche & Sileno & $=$ Sileno. Rivista di studi classici e cristiani \\
\hline $\begin{array}{l}\mathrm{RStBiz} \\
\mathrm{RStCl}\end{array}$ & $\begin{array}{l}=\text { Rivista di studi bizantini e neoellenici } \\
\text { = Rivista di studi classici }\end{array}$ & Sintria & $\begin{array}{l}=\text { Sintria. Revista de estudos de arqueologia, } \\
\text { arte e etnografia }\end{array}$ \\
\hline RStFen & $=$ Rivista di studi fenici & SlovA & =Slovenská archeológia \\
\hline RStLig & $=$ Rivista di studi liguri & SlovNum & =Slovenská numizmatika \\
\hline RStMarch & $=$ Rivista di studi marchigiani & SMEA & =Studi micenei ed egeo-anatolici \\
\hline
\end{tabular}




\begin{tabular}{|c|c|c|c|}
\hline SocGeoAOran & $\begin{aligned}= & \text { (Bulletin trimestriel de la) Société de } \\
& \text { géographie et d'archéologie (de la } \\
& \text { province) d'Oran }\end{aligned}$ & $\begin{array}{l}\text { StUrbin } \\
\text { Sumer }\end{array}$ & $\begin{array}{l}=\text { Studi urbinati, } \mathrm{B} \text {. Scienze umane e sociali } \\
=\text { Sumer }\end{array}$ \\
\hline SoobErmit & $\begin{array}{l}=\text { Soobščenija Gosudarstvennogo ordena } \\
\text { Lenina Ėrmitaža }\end{array}$ & Sylva Mala & $\begin{aligned}= & \text { Sylva Mala. Bollettino del Centro di studi } \\
& \text { archeologici di Boscoreale, Boscotrecase e } \\
& \text { Trecase }\end{aligned}$ \\
\hline SovA & $=$ Sovetskaja archeologija & SymbOslo & = Symbolae Osloenses \\
\hline \multirow[t]{2}{*}{ SpNov } & \multirow{2}{*}{$\begin{aligned} &= \text { Specimina nova dissertationum ex } \\
& \text { Instituto historico Universitatis } \\
& \text { Quinqueecclesiensis de Iano Pannonio } \\
& \text { nominatae }\end{aligned}$} & Syria & $\begin{array}{l}=\text { Syria. Revue d'art oriental et } \\
\text { d'archéologie }\end{array}$ \\
\hline & & \multirow[t]{4}{*}{ SyrMesopSt } & \multirow[t]{4}{*}{$=$ Syro-Mesopotamian Studies } \\
\hline Spoletium & $=$ Spoletium. Rivista di arte, storia, cultura & & \\
\hline StA & $=$ Studia archaeologica & & \\
\hline StädelJb & $=$ Städel-Jahrbuch & & \\
\hline Stadion & $\begin{array}{l}=\text { Stadion. Zeitschrift für Geschichte des } \\
\text { Sports und der Körperkultur }\end{array}$ & Talanta & $\begin{aligned}= & \text { Tád } \alpha v \tau \alpha . \text { Proceedings of the Dutch } \\
& \text { Archaeological and Historical Society }\end{aligned}$ \\
\hline StAeg & $=$ Studia aegyptiaca. Budapest & Taras & $=$ Taras. Rivista di archeologia \\
\hline StAltägKul & $=$ Studien zur altägyptischen Kultur & Teiresias & $\begin{aligned}= & \text { Teiresias. A review and continuing } \\
& \text { bibliography of Boiotian studies }\end{aligned}$ \\
\hline StAnt & = Studi di antichità. Università di Lecce & TelAvivJA & $=$ Tel Aviv. Journal of the Tel Aviv \\
\hline $\begin{array}{l}\text { Starinar } \\
\text { StAWarsz }\end{array}$ & $\begin{array}{l}=\text { Starinar. Arheološki institut, Beograd } \\
=\text { Studia archeologiczne. Uniwersytet }\end{array}$ & & University Institute of Archaeology \\
\hline & Warszawski, Instytut archeologii & TerraAntBalc & $\begin{aligned}= & \text { Acta Associationis internationalis Terra } \\
& \text { Antiqua Balcanica }\end{aligned}$ \\
\hline Stbirranc & $\begin{array}{l}=\text { Jtudium bidicum tranciscanum. Liber } \\
\text { annuus }\end{array}$ & Teruel & $=$ Teruel. Instituto de estudios turolenses \\
\hline StCerclstorV & $\begin{aligned}= & \text { Studii şi cercetări de istorie veche şi } \\
& \text { arheologie }\end{aligned}$ & TextilAnc & $\begin{array}{l}=\text { Textiles anciens. Bulletin du Centre } \\
\text { international d'étude des textiles anciens }\end{array}$ \\
\hline StCercNum & = Studii şi cercetări de numismatică & TheolRu & $=$ Theologische Rundschau \\
\hline $\mathrm{StCl}$ & $\begin{aligned}= & \text { Studii clasice. Societatea de studii clasice } \\
& \text { din Republica socialistă Romănia }\end{aligned}$ & Thessalika & $=\Theta \varepsilon \sigma \sigma \alpha \lambda \iota x \alpha$ \\
\hline $\mathrm{StClOr}$ & $=$ Studi classici e orientali & Thessalonike & $=\mathrm{H} \Theta \varepsilon \sigma \sigma \alpha \lambda \sigma v i x \eta$ \\
\hline StDocA & $=$ Studi e documenti di archeologia & Thracia & $=$ Thracia \\
\hline StDocHistIur & $=$ Studia et documenta historiae et iuris & Thraco-Dacica & $=$ Thraco-Dacica \\
\hline StEbla & $=$ Studi eblaiti & ThrakChron & 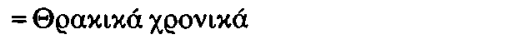 \\
\hline StEgAntPun & $=$ Studi di egittologia e di antichità puniche & Tibiscus & $\begin{aligned}= & \text { Tibiscus. Istorie, arheologie. Muzeul } \\
& \text { Banatului Timişoara }\end{aligned}$ \\
\hline SteMat & $\begin{aligned}= & \text { Studi e materiali. Sopintendenza ai beni } \\
& \text { archeologici per la Toscana }\end{aligned}$ & TiLeon & $=$ Tierras de León \\
\hline StEpigrLing & $\begin{aligned}= & \text { Studi epigrafici e linguistici sul Vicino } \\
& \text { Oriente antico }\end{aligned}$ & TourOrl & $\begin{array}{l}=\text { La Tour de l'Orle-d'Or, Semur-en- } \\
\text { Auxois }\end{array}$ \\
\hline StEtr & $=$ Studi etruschi & TrabAn & $=$ Trabalhos de antropologia e etnologia \\
\hline StGenu & $=$ Studi genuensi & TrabPr & $=$ Trabajos de prehistoria \\
\hline \multirow{2}{*}{\multicolumn{2}{|c|}{$\begin{aligned} \text { StiftHamburgKuSamml }= & \text { Stiftung zur Förderung der } \\
& \text { Hamburgischen Kunstsammlungen. } \\
& \text { Erwerbungen }\end{aligned}$}} & Traditio & $\begin{array}{l}=\text { Traditio. Studies in ancient and medieval } \\
\text { history, thought and religion }\end{array}$ \\
\hline StItFilCl & & TransactAmPhilAss & $\begin{aligned}= & \text { Transactions and Proceedings of the } \\
& \text { American Philological Association }\end{aligned}$ \\
\hline $\begin{array}{l}\text { StLatIt } \\
\text { StMagreb }\end{array}$ & $\begin{array}{l}=\text { Studi latini e italiani } \\
=\text { Studi magrebini }\end{array}$ & TransactLond & $\begin{aligned}= & \text { Transactions of the London and } \\
& \text { Middlesex Archaeological Society }\end{aligned}$ \\
\hline StMatStorRel & $=$ Studi magrebin 1 Studi e materiali di storia delle religioni & TravMem & $\begin{array}{l}=\text { Travaux et mémoires. Centre de recherche } \\
\text { d'histoire et civilisation byzantine. Paris }\end{array}$ \\
\hline $\begin{array}{l}\text { StOliv } \\
\text { StOr }\end{array}$ & $\begin{array}{l}=\text { Studia Oliveriana } \\
=\text { Studia orientalia, Helsinki }\end{array}$ & TravToulouse & $\begin{aligned}= & \text { Travaux de l'Institut d'art préhistorique, } \\
& \text { Université de Toulouse - Le Mirail }\end{aligned}$ \\
\hline $\mathrm{StP}$ & $=$ Studia papyrologica & TreMonet & $=$ Trésors monétaires \\
\hline StrennaRom & $=$ Strenna dei romanist $i$ & TrudyErmit & = Trudy Gosudarstvennogo Ėrmitaža \\
\hline StRom & $=$ Studi romani & $\operatorname{TrWPr}$ & $=$ Trierer Winckelmannsprogramme \\
\hline StRomagn & $=$ Studi romagnoli & \multirow[t]{2}{*}{$\operatorname{Tr} Z$} & \multirow{2}{*}{$\begin{aligned}= & \text { Trierer Zeitschrift für Geschichte und } \\
& \text { Kunst des Trierer Landes und seiner } \\
& \text { Nachbargebiete }\end{aligned}$} \\
\hline $\begin{array}{l}\text { StSalent } \\
\text { StSard }\end{array}$ & $\begin{array}{l}=\text { Studi salentini } \\
=\text { Studi sardi }\end{array}$ & & \\
\hline StStorRel & $=$ Studi storico-religiosi & TürkAD & $=$ Türk arkeoloji dergisi \\
\hline StTardoant & $=$ Studi tardoantichi & Tuy & $=$ Tuy. Museo y archivo histórico diocesano \\
\hline StTrentStor & $\begin{aligned}= & \text { Studi trentini di scienze storiche. Sezione } \\
& \text { seconda }\end{aligned}$ & Tyche & $\begin{aligned}= & \text { Tyche. Beiträge zur Alten Geschichte, } \\
& \text { Papyrologie und Epigraphik }\end{aligned}$ \\
\hline
\end{tabular}


UgaritF

Uni-HH-Forschung

L'Urbe

UrSchw

VarSpom
Vekove
VenArt
VerAmstMeded
Verbanus
VesDrevIstor
VeteraChr
Vichiana
VicOr
VigChr
VisRel
VizVrem
VjesAMuzZagreb
VjesDal

VjesDal
= Varstvo spomenikov

= Vekove. Dvumesečno spisanie. Bălgarsko istoričesko družestvo

= Venezia arti. Bollettino del Dipartimento di storia e critica delle arti dell'Università di Venezia.

$=$ Vereniging van vrienden Allard Pierson museum Amsterdam. Mededelingenblad

= Verbanus. Rassegna per la cultura, l'arte, la storia del lago

$=$ Vestnik drevnej istorii

= Vetera Christianorum

= Vichiana. Rassegna di studi filologici e storici

$=$ Vicino Oriente

$=$ Vigiliae christianae

= Visible Religion

= Vizantijskij vremennik

$=$ Vjesnik Arheološkog muzeja u Zagrebu

$=$ Vjesnik $\mathrm{za}$ arheologiju i historiju

dalmatinsku. Bulletin d'archéologie et d'histoire dalmates.

= Wad-al-Hayara. Revista de estudios de la Institución provincial de cultura

"Marqués de Santillana“ de Guadalajara

WeltGesch

WiadA

WissMBosn

WissZBerl

WissZHalle

WissZJena

WissZRostock

WO

WorldA

WSt

WürzbJb

WZKM

$=$ Wiadomści archeologiczne. Bulletin archéologique polonais

$=$ Wissenschaftliche Mitteilungen des Bosnisch-Herzegowinischen Landesmuseums, A. Archäologie

$=$ Wissenschaftliche Zeitschrift der Humboldt-Universität zu Berlin. Gesellschafts- und sprachwissenschaftliche Reihe

$=$ Wissenschaftliche Zeitschrift. MartinLuther-Universität Halle-Wittenberg

$=$ Wissenschaftliche Zeitschrift der Friedrich-Schiller-Universität Jena

$=$ Wissenschaftliche Zeitschrift der Universität Rostock

$=$ Die Welt des Orients

$=$ World Archaeology

$=$ Wiener Studien

= Würzburger Jahrbücher für die Altertumswissenschaft

$=$ Wiener Zeitschrift für die Kunde des
Xenia

= Xenia. Semestrale di antichità

YaleCISt

YaleUnivB

$=$ Yale Classical Studies

= Yale University Art Gallery Bulletin

ZA

ZÄS

ZAW

ZborMuzBeograd ZborRadBeograd

ZborZadar

ZDMG

ZDPV

Zephyrus

ZEthn

ZfA

ŽivaAnt

ZKuGesch

ZNW

ZPE

ZSav

ZSchwA

ZVerglSprF
$=$ Zeitschrift für Assyriologie und vorderasiatische Archäologie

= Zeitschrift für ägyptische Sprache und Altertumskunde

$=$ Zeitschrift für die alttestamentliche Wissenschaft

=Zbornik Narodnog muzeja Beograd

= Zbornik radova Vizantološkog instituta. Recueil des travaux de l'Institut d'études byzantines, Beograd

= Zbornik Instituta za historijske nauke u Zadru

= Zeitschrift der Deutschen

Morgenländischen Gesellschaft

$=$ Zeitschrift des Deutschen Palästina-

Vereins

=Zephyrus

$=$ Zeirschrift für Ethnologie

$=$ Zeitschrift für Archäologie

= Živa antika. Antiquité vivante

$=$ Zeitschrift für Kunstgeschichte

$=$ Zeitschrift für die neutestamentliche Wissenschaft und die Kunde der älteren Kirche

$=$ Zeitschrift für Papyrologie und Epigraphik

$=$ Zeitschrift der Savigny-Stiftung für Rechtsgeschichte. Romanistische Abteilung

$=$ Zeitschrift für schweizerische Archäologie und Kunstgeschichte

$=$ Zeitschrift für vergleichende Sprachforschung Morgenlandes 